\title{
A New Approach to the Fuzzification of Convex Structures
}

\author{
Fu-Gui Shi and Zhen-Yu Xiu \\ School of Mathematics and Statistics, Beijing Institute of Technology, Beijing 100081, China \\ Correspondence should be addressed to Zhen-Yu Xiu; xyz198202@163.com
}

Received 26 April 2014; Revised 19 June 2014; Accepted 27 June 2014; Published 31 August 2014

Academic Editor: Jin Liang

Copyright (C) 2014 F.-G. Shi and Z.-Y. Xiu. This is an open access article distributed under the Creative Commons Attribution License, which permits unrestricted use, distribution, and reproduction in any medium, provided the original work is properly cited.

\begin{abstract}
A new approach to the fuzzification of convex structures is introduced. It is also called an $M$-fuzzifying convex structure. In the definition of $M$-fuzzifying convex structure, each subset can be regarded as a convex set to some degree. An $M$-fuzzifying convex structure can be characterized by means of its $M$-fuzzifying closure operator. An $M$-fuzzifying convex structure and its $M$-fuzzifying closure operator are one-to-one corresponding. The concepts of $M$-fuzzifying convexity preserving functions, substructures, disjoint sums, bases, subbases, joins, product, and quotient structures are presented and their fundamental properties are obtained in $M$-fuzzifying convex structure.
\end{abstract}

\section{Introduction and Preliminaries}

Convexity theory has been accepted to be of increasing importance in recent years in the study of extremum problems in many areas of applied mathematics. The concept of convexity which was mainly defined and studied in $R^{n}$ in the pioneering works of Newton, Minkowski, and others as described in [1] now finds a place in several other mathematical structures such as vector spaces, posets, lattices, metric spaces, graphs, and median algebras. This development is motivated not only by the need for an abstract theory of convexity generalizing the classical theorems in $R^{n}$ due to Helly, Caratheodory, and so forth but also by the necessity to unify geometric aspects of all these mathematical structures. Some more details can be found in [2].

In 1994, Rosa presented the notion of fuzzy convex structures in $[3,4]$. In 2009, Maruyama generalized it to $M$ fuzzy setting in [5]. A fuzzy convex structure is a pair of $(X, \mathscr{C})$ in which $\mathscr{C}$ is a crisp subset of the set of $M$-fuzzy subsets of a nonempty set $X$ satisfying certain set of axioms.

In this paper, from a completely different point of view, we introduce the notion of $M$-fuzzifying convexity on a nonempty set $X$ by means of a mapping $\mathscr{C}: 2^{X} \rightarrow M$ satisfying three axioms, where $M$ is a complete lattice and $\mathbf{2}^{X}$ is the set of all subsets of $X$. Thus, each subset of $X$ can be regarded as a convex set to some degree.
Throughout this paper, $\left(M, \bigvee, \bigwedge,{ }^{\prime}\right)$ denotes a complete lattice with an order-reversing involution'. The smallest element (or zero element) and the largest element (or unit element) in $M$ are denoted by $\perp$ and T, respectively. $2^{X}$, resp, $2_{\text {fin }}^{X}$, denotes the collection of all subsets, respectively, all finite subsets of a nonempty set $X$.

The binary relation $\prec$ in $M$ is defined as follows: for $a, b \in$ $M, a<b$ if and only if for every subset $D \subseteq M$, the relation $b \leq \sup D$ always implies the existence of $d \in D$ with $a \leq d$ [6]. $\{a \in M: a<b\}$ is called the greatest minimal family of $b$ in the sense of [7], denoted by $\beta(b)$. Moreover, for $b \in M$, we define $\alpha(b)=\left\{a \in M: b \prec{ }^{\mathrm{op}} a\right\}$.

In a completely distributive lattice $M$ with an orderreversing involution', there exist $\alpha(b)$ and $\beta(b)$ for each $b \in$ $M, b=\bigvee \beta(b)=\bigwedge \alpha(b)$, and $a \prec b \Leftrightarrow b^{\prime}{ }^{\mathrm{op}} a^{\prime}$ (see [7]).

Theorem 1 (see [7]). If $M$ is completely distributive, then for a subfamily $\left\{a_{i}: i \in \Omega\right\}$ of $M$, one has

(1) $\alpha\left(\bigwedge_{i \in \Omega} a_{i}\right)=\bigcup_{i \in \Omega} \alpha\left(a_{i}\right)$; that is, $\alpha$ is an $\Lambda-\bigcup$ mapping.

(2) $\beta\left(\bigvee_{i \in \Omega} a_{i}\right)=\bigcup_{i \in \Omega} \beta\left(a_{i}\right)$; that is, $\beta$ is a union-preserving mapping. 
For a complete lattice $M, A \in M^{X}$, and $a \in M$, we use the following notation:

$$
A_{[a]}=\{x \in X: A(x) \geq a\}
$$

Suppose that $M$ is completely distributive and we define

$$
A^{[a]}=\{x \in X: a \notin \alpha(A(x))\}
$$

Some properties of these cut sets can be found in [8-13].

Theorem 2 (see $[8,10,13])$. If $M$ is completely distributive, then for each $M$-fuzzy set $A$ in $M^{X}$, one has

(1) $A=\bigvee_{a \in M}\left(a \wedge A_{[a]}\right)=\bigwedge_{a \in M}\left(a \vee A^{[a]}\right)$.

(2) $\forall a \in M, A_{[a]}=\bigcap_{b \in \beta(a)} A_{[b]}$.

(3) $\forall a \in M, A^{[a]}=\bigcap_{a \in \alpha(b)} A^{[b]}$.

Lemma 3. Let $M$ be a completely distributive lattice and let $p, q \in M$. Then the following conditions are equivalent:

(1) $p \leq q$.

(2) $\forall a \in M$; if $a \leq p$ then $a \leq q$.

(3) $\forall a \in \beta(T)$; if $a \leq p$ then $a \leq q$.

(4) $\forall a \in \alpha(\perp)$; if $a \notin \alpha(p)$ then $a \notin \alpha(q)$.

Proof. It is easy to know that (1) $\Leftrightarrow(2),(2) \Rightarrow(3)$, and (2) $\Rightarrow$ (4) hold. Next we prove (3) $\Rightarrow(2)$ and (4) $\Rightarrow(2)$.

(3) $\Rightarrow(2) \forall a \in M$; if $a \leq p$, then $\vee \beta(a)=a \leq p$. Hence, $\forall b \in \beta(a) \subseteq \beta(T), b \leq p$. By (3), $b \leq q$. Then $a=\vee \beta(a) \leq q$.

$(4) \Rightarrow(2) \forall a \in M$; if $a \leq p$ then $\alpha(p) \subseteq \alpha(a) \subseteq \alpha(\perp)$. $\forall b \in \alpha(\perp)$; if $b \notin \alpha(a)$ then $b \notin \alpha(p)$. By $(4), b \notin \alpha(q)$. Hence, $\alpha(a) \cap \alpha(\perp) \supseteq \alpha(q) \cap \alpha(\perp)$ and thus $\alpha(a) \supseteq \alpha(q)$. So $a=\wedge \alpha(a) \leq \wedge \alpha(q)=q$.

Definition 4 (see [2]). A subset $\mathscr{C}$ of $2^{X}$ is called a convexity if it satisfies the following three conditions:

(C1) $\emptyset, X \in \mathscr{C}$;

(C2) if $\left\{A_{i}: i \in \Omega\right\} \subseteq \mathscr{C}$ is nonempty, then $\bigcap_{i \in \Omega} A_{i} \in \mathscr{C}$;

(C3) if $\left\{A_{i}: i \in \Omega\right\} \subseteq \mathscr{C}$ is nonempty and totally ordered by inclusion, then $\bigcup_{i \in \Omega} A_{i} \in \mathscr{C}$.

The fuzzy sets in $\mathscr{C}$ are called convex sets, and the pair $(X, \mathscr{C})$ is called a convex structure.

If $\mathscr{C}$ satisfies $(\mathrm{C} 1)$ and $(\mathrm{C} 2)$, then $(X, \mathscr{C})$ is called a closure structure.

Theorem 5 (see $[2,14])$. Let $(X, \mathscr{C})$ be a convex structure and $\emptyset \neq Y \subseteq X$. For $A \in \mathscr{C}, \operatorname{co}(A \cap Y) \cap Y=A \cap Y$, where co is the hull operator of $(X, \mathscr{C})$.

Definition 6 (see [2]). A closure operator $\mathrm{cl}$ on $X$ is domain finite (or algebraic) provided for each $A \subseteq X$ and for each $p \in \operatorname{cl}(A)$ there is a finite set $F \subseteq A$ with $p \in \operatorname{cl}(F)$.
Theorem 7 (see $[2])$. Let $(X, \mathscr{C})$ be a closure structure. Then the following conditions are equivalent:

(1) $\mathscr{C}$ is a convexity on $X$;

(2) the closure operator of $\mathscr{C}$ is domain finite;

(3) $\mathscr{C}$ is stable for updirected union; that is, if $\left\{A_{i}: i \in\right.$ $\Omega\} \subseteq 2^{X}$ is an updirected set then $\bigcup_{i \in \Omega} A_{i} \in \mathscr{C}$.

In 1994, Rosa presented the notion of fuzzy convex structures in [3, 4]. In 2009, Maruyama generalized it to $M$ fuzzy setting in [5] as follows.

Definition 8 (see [5]). For a nonempty set $X$ and a subset $\mathscr{C}$ of $M^{X},(X, \mathscr{C})$ is called a fuzzy convex structure if and only if $(X, \mathscr{C})$ satisfies the following conditions:

(MC1) $\chi_{\emptyset}, \chi_{X} \in \mathscr{C}$;

(MC2) if $\left\{A_{i}: i \in \Omega\right\} \subseteq \mathscr{C}$ is nonempty, then $\bigwedge_{i \in \Omega} A_{i} \in \mathscr{C}$;

(MC3) if $\left\{A_{i}: i \in \Omega\right\} \subseteq \mathscr{C}$ is nonempty and totally ordered by inclusion, then $\bigvee_{i \in \Omega} A_{i} \in \mathscr{C}$.

Based on papers $[15,16]$, we can obtain the following definitions and theorems.

Definition 9 (see $[15,16])$. A mapping $\mathscr{C}: 2^{X} \rightarrow M$ is called an $M$-fuzzifying closure system if it satisfies the following conditions:

(MYC) $\mathscr{C}(X)=\mathrm{T}$;

(MYC2) if $\left\{A_{i}: i \in \Omega\right\} \subseteq \mathbf{2}^{X}$ is nonempty, then $\mathscr{C}\left(\bigcap_{i \in \Omega} A_{i}\right) \geq \bigwedge_{i \in \Omega} \mathscr{C}\left(A_{i}\right)$.

A pair $(X, \mathscr{C})$ is called an $M$-fuzzifying closure system space provided that $\mathscr{C}$ is an $M$-fuzzifying closure system on $X$.

Definition 10 (see $[15,16])$. An $M$-fuzzifying closure operator on $X$ is a mapping $\mathrm{cl}: 2^{X} \rightarrow M^{X}$ satisfying the following conditions:

(CL1) $\operatorname{cl}(A)(x)=\top$ for every $x \in A$;

(CL2) $A \subseteq B \Rightarrow \mathrm{cl}(A) \leq \mathrm{cl}(B)$;

(CL3) $\operatorname{cl}(A)(x)=\bigwedge_{x \notin B \supseteq A} \bigvee_{y \notin B} \operatorname{cl}(B)(y)$.

Theorem 11 (see $[15,16])$. Let $\mathrm{cl}$ be an $M$-fuzzifying closure operator on $X$. Define a mapping $\mathscr{C}_{\mathrm{cl}}: 2^{X} \rightarrow M$ by

$$
\forall A \subseteq X, \quad \mathscr{C}_{\mathrm{cl}}(A)=\bigwedge_{x \notin A}(\mathrm{cl}(A)(x))^{\prime} .
$$

Then $\mathscr{C}_{\mathrm{cl}}$ is an $M$-fuzzifying closure system.

Theorem 12 (see $[15,16])$. Let $(X, \mathscr{C})$ be an M-fuzzifying closure system space. Define $\mathrm{cl}_{\mathscr{C}}: \mathbf{2}^{X} \rightarrow M^{X}$ by

$$
\begin{array}{r}
\forall x \in X, \quad \forall A \subseteq X, \\
\operatorname{cl}_{\mathscr{C}}(A)(x)=\bigwedge_{x \notin B \supseteq A}(\mathscr{C}(B))^{\prime} .
\end{array}
$$

Then $\mathrm{cl}_{\mathscr{C}}$ is an M-fuzzifying closure operator on $X$. 
Theorem 13 (see $[15,16])$. Let $(X, \mathscr{C})$ be an M-fuzzifying closure system space and let $\mathrm{cl}$ be an M-fuzzifying closure operator on $X$. Then $\mathrm{cl}_{\mathscr{C}_{\mathrm{cl}}}=\mathrm{cl}$ and $\mathscr{C}_{\mathrm{cl}_{\mathscr{G}}}=\mathscr{C}$.

Definition 14 (see [17]). A fuzzy vector space is a pair $(V, \mu)$, where $V$ is a vector space on a totally ordered field $\mathbb{K}$ and $\mu$ : $V \rightarrow[0,1]$ is a mapping satisfying $\mu(k x+l y) \geq \mu(x) \wedge \mu(y)$ for any $x, y \in V, k, l \in \mathbb{K}$. If $(V, \mu)$ is a fuzzy vector space, then for each $a \in(0,1], \mu_{[a]}$ is a subspace of $V$.

\section{M-Fuzzifying Convex Structures}

In this section, from a completely different point of view, we introduce the notion of $M$-fuzzifying convexity on a nonempty set $X$ by means of a mapping $\mathscr{C}: 2^{X} \rightarrow M$. Thus, each subset of $X$ can be regarded as a convex set to some degree.

Definition 15. A mapping $\mathscr{C}: \mathbf{2}^{X} \rightarrow M$ is called an $M$-fuzzifying convexity on $X$ if it satisfies the following conditions:

$(\mathrm{MYC1}) \mathscr{C}(\emptyset)=\mathscr{C}(X)=\mathrm{T}$

(MYC2) if $\left\{A_{i}: i \in \Omega\right\} \subseteq \mathbf{2}^{X}$ is nonempty, then $\mathscr{C}\left(\bigcap_{i \in \Omega} A_{i}\right) \geq \bigwedge_{i \in \Omega} \mathscr{C}\left(A_{i}\right)$;

(MYC3) if $\left\{A_{i}: i \in \Omega\right\} \subseteq \mathbf{2}^{X}$ is nonempty and totally ordered by inclusion, then $\mathscr{C}\left(\bigcup_{i \in \Omega} A_{i}\right) \geq \bigwedge_{i \in \Omega} \mathscr{C}\left(A_{i}\right)$.

If $\mathscr{C}$ is an $M$-fuzzifying convexity on $X$, then the pair $(X, \mathscr{C})$ is called an $M$-fuzzifying convex structure. For $A \in$ $2^{X}, \mathscr{C}(A)$ can be regarded as the degree to which $A$ is a convex set. When $M=I=[0,1]$, an $M$-fuzzifying convex structure is called a fuzzifying convex structure for short.

If $\mathscr{C}$ satisfies (MYC1) and (MYC2), then $(X, \mathscr{C})$ is called an $M$-fuzzifying closure structure.

Remark 16. (1) We can see that an $M$-fuzzifying closure structure $(X, \mathscr{C})$ is exactly an $M$-fuzzifying closure system space $(X, \mathscr{C})$ with $\mathscr{C}(\emptyset)=T$.

(2) By Theorems 11 and 12 , we can verify that there is a one-to-one correspondence between $M$-fuzzifying closure structures and $M$-fuzzifying closure operators with (CLO), where

$($ CL0) $\operatorname{cl}(\emptyset)(x)=\perp$ for every $x \in X$.

Therefore, we can obtain the $M$-fuzzifying closure operator induced by an $M$-fuzzifying closure structure that satisfies (CL0)-(CL3).

Example 17 (see $[16,18])$. Let $X$ be a universe of discourse. A mapping $\mathscr{T}: 2^{X} \rightarrow I$ is called a fuzzifying topology on $X$ if it satisfies the following conditions:

$$
\begin{aligned}
& \text { (FOA1) } \mathscr{T}(\emptyset)=\mathscr{T}(X)=1 ; \\
& \text { (FOA2) } A_{1}, A_{2} \in 2^{X}, \mathscr{T}\left(A_{1} \cap A_{2}\right) \geq \mathscr{T}\left(A_{1}\right) \wedge \mathscr{T}\left(A_{2}\right) ; \\
& \text { (FOA3) } \forall\left\{A_{j}\right\}_{j \in J} \subseteq 2^{X}, \mathscr{T}\left(\bigcup_{j \in J} A_{j}\right) \geq \bigwedge_{j \in J} \mathscr{T}\left(A_{j}\right) .
\end{aligned}
$$

And $(X, \mathscr{T})$ is called a fuzzifying topological space. Furthermore, if a fuzzifying topology $\mathscr{T}$ satisfies

(SOA2) $\forall\left\{A_{j}\right\}_{j \in J} \subseteq \mathbf{2}^{X}, \mathscr{T}\left(\bigcap_{j \in J} A_{j}\right) \geq \bigwedge_{j \in J} \mathscr{T}\left(A_{j}\right)$, then $\mathscr{T}$ is called a saturated fuzzifying topology and $(X, \mathscr{T})$ is called an Alexandroff fuzzifying topological space.

We can see that an Alexandroff fuzzifying topological space $(X, \mathscr{T})$ is a fuzzifying convex structure.

Example 18. Let $(V, \mu)$ be a fuzzy vector space. Define a mapping $\mathscr{C}_{\mu}: 2^{V} \rightarrow[0,1]$ by

$$
\mathscr{C}_{\mu}(A)=\bigvee\left\{a \in(0,1]: A \in \mathscr{C}_{a}\right\},
$$

where $\mathscr{C}_{a} \subseteq 2^{\mu_{[a]}}$ is the standard convexity of $\mu_{[a]}$ for each $a \in(0,1]$; that is, $A \in \mathscr{C}_{a}$ if and only if for all $x, y \in A$ and for each $t \in \mathbb{K}$ with $0 \leq t \leq 1, t \cdot x+(1-t) y \in A$. Then $\left(V, \mathscr{C}_{\mu}\right)$ is a fuzzifying convex structure. It is easy to see that $\mathscr{C}_{\mu}$ satisfies (MYC1). Now we prove that $\mathscr{C}_{\mu}$ satisfies (MYC2) and (MYC3).

(MYC2) Suppose that $\left\{A_{i}: i \in \Omega\right\} \subseteq \mathbf{2}^{X}$ is nonempty and take any $b<\bigwedge_{i \in \Omega} \mathscr{C}_{\mu}\left(A_{i}\right)$. Then for each $i \in \Omega, \mathscr{C}_{\mu}\left(A_{i}\right)>b$. By the definition of $\mathscr{C}_{\mu}$, we know that, for each $i \in \Omega$, there exists $a_{i}>b$ such that $A_{i} \in \mathscr{C}_{a_{i}} \subseteq \mathscr{C}_{b}$. Since $\mathscr{C}_{b}$ is the standard convexity of $\mu_{[b]}, \bigcap_{i \in \Omega} A_{i} \in \mathscr{C}_{b}$. This shows that $\mathscr{C}_{\mu}\left(\bigcap_{i \in \Omega} A_{i}\right) \geq b$. By the arbitrariness of $b$, we obtain that $\mathscr{C}_{\mu}\left(\bigcap_{i \in \Omega} A_{i}\right) \geq \bigwedge_{i \in \Omega} \mathscr{C}_{\mu}\left(A_{i}\right)$.

(MYC3) Suppose that $\left\{A_{i}: i \in \Omega\right\} \subseteq 2^{X}$ is nonempty and totally ordered by inclusion, and take any $b<\bigwedge_{i \in \Omega} \mathscr{C}_{\mu}\left(A_{i}\right)$. Then for each $i \in \Omega, \mathscr{C}_{\mu}\left(A_{i}\right)>b$. By the definition of $\mathscr{C}_{\mu}$, we know that, for each $i \in \Omega$, there exists $a_{i}>b$ such that $A_{i} \in \mathscr{C}_{a_{i}} \subseteq \mathscr{C}_{b}$. Since $\mathscr{C}_{b}$ is the standard convexity of $\mu_{[b]}$, $\bigcup_{i \in \Omega} A_{i} \in \mathscr{C}_{b}$. This shows that $\mathscr{C}_{\mu}\left(\bigcup_{i \in \Omega} A_{i}\right) \geq b$. By the arbitrariness of $b$, we obtain that $\mathscr{C}_{\mu}\left(\bigcup_{i \in \Omega} A_{i}\right) \geq \bigwedge_{i \in \Omega} \mathscr{C}_{\mu}\left(A_{i}\right)$.

Example 19. Let $(X, \mathscr{C})$ be a crisp convex structure. Define $\chi_{\mathscr{C}}: \mathbf{2}^{X} \rightarrow[0,1]$ by

$$
\chi_{\mathscr{C}}(A)= \begin{cases}1, & A \in \mathscr{C} ; \\ 0, & A \notin \mathscr{C} .\end{cases}
$$

Then it is easy to prove that $\left(X, \chi_{\mathscr{C}}\right)$ is a fuzzifying convex structure.

Example 20. Let $X$ be a nonempty set and let $\mathscr{C}: \mathbf{2}^{X} \rightarrow[0,1]$ be a mapping defined by

$$
\mathscr{C}(A)= \begin{cases}1, & A \in\{\emptyset, X\} ; \\ 0.5, & A \notin\{\emptyset, X\} .\end{cases}
$$

Then it is easy to prove that $(X, \mathscr{C})$ is a fuzzifying convex structure. If $A \in 2^{X}$ with $A \notin\{\emptyset, X\}$, then 0.5 is the degree to which $A$ is a convex set.

Theorem 21. Let $\mathscr{C}: \mathbf{2}^{X} \rightarrow M$ be a mapping. Then $(X, \mathscr{C})$ is an $M$-fuzzifying convex structure if and only if, for each a $\epsilon$ $M \backslash\{\perp\},\left(X, \mathscr{C}_{[a]}\right)$ is a convex structure.

Proof. This is straightforward.

Theorem 22. If $M$ is completely distributive, then a mapping $\mathscr{C}: \mathbf{2}^{X} \rightarrow M$ is an M-fuzzifying convexity if and only if, for each $a \in \alpha(\perp), \mathscr{C}^{[a]}$ is a convexity. 
Proof. Sufficiency. (MYC1) For each $a \in \alpha(\perp), \emptyset, X \in \mathscr{C}^{[a]}$. We have $\mathscr{C}(\emptyset)=\mathscr{C}(X)=\mathrm{T}$.

(MYC2) Let $\left\{A_{i}: i \in \Omega\right\} \subseteq 2^{X}$ be nonempty and for $a \in \alpha(\perp), a \notin \alpha\left(\bigwedge_{i \in \Omega} \mathscr{C}\left(A_{i}\right)\right)$. Thus, $a \notin \bigcup_{i \in \Omega} \alpha\left(\mathscr{C}\left(A_{i}\right)\right)$. We know that $a \notin \alpha\left(\mathscr{C}\left(A_{i}\right)\right)$ and then $A_{i} \in \mathscr{C}^{[a]}$ for each $i \in \Omega$. Since for each $a \in \alpha(\perp), \mathscr{C}^{[a]}$ is a convexity, $\bigcap_{i \in \Omega} A_{i} \in \mathscr{C}^{[a]}$; that is, $a \notin \alpha\left(\mathscr{C}\left(\bigcap_{i \in \Omega} A_{i}\right)\right)$. Therefore, by the arbitrariness of $a$ and Lemma $3, \mathscr{C}\left(\bigcap_{i \in \Omega} A_{i}\right) \geq \bigwedge_{i \in \Omega} \mathscr{C}\left(A_{i}\right)$.

(MYC3) Let $\left\{A_{i}: i \in \Omega\right\} \subseteq 2^{X}$ be nonempty and totally ordered by inclusion and let $a \notin \alpha\left(\bigwedge_{i \in \Omega} \mathscr{C}\left(A_{i}\right)\right)$ for $a \in \alpha(\perp)$. Thus, $a \notin \bigcup_{i \in \Omega} \alpha\left(\mathscr{C}\left(A_{i}\right)\right)$. We know that $a \notin$ $\alpha\left(\mathscr{C}\left(A_{i}\right)\right)$ and then $A_{i} \in \mathscr{C}^{[a]}$ for each $i \in \Omega$. Since for each $a \in \alpha(\perp), \mathscr{C}^{[a]}$ is a convexity, $\bigcup_{i \in \Omega} A_{i} \in \mathscr{C}^{[a]}$; that is, $a \notin \alpha\left(\mathscr{C}\left(\bigcup_{i \in \Omega} A_{i}\right)\right)$. Therefore, by the arbitrariness of $a$ and Lemma $3, \mathscr{C}\left(\bigcup_{i \in \Omega} A_{i}\right) \geq \bigwedge_{i \in \Omega} \mathscr{C}\left(A_{i}\right)$.

Necessity. Suppose that $\mathscr{C}: \mathbf{2}^{X} \rightarrow M$ is an $M$-fuzzifying convexity and $a \in \alpha(\perp)$. Now we prove that $\mathscr{C}^{[a]}$ is a convexity.

(C1) By $\mathscr{C}(\emptyset)=\mathscr{C}(X)=\top$ and $\alpha(T)=\emptyset$, we know that $a \notin \alpha(\mathscr{C}(\emptyset))$ and $a \notin \alpha(\mathscr{C}(X))$. This implies that $\emptyset, X \in \mathscr{C}^{[a]}$.

(C2) If $\left\{A_{i}: i \in \Omega\right\} \subseteq \mathscr{C}^{[a]}$, then for all $i \in \Omega$, $a \notin \alpha\left(\mathscr{C}\left(A_{i}\right)\right)$. Hence, $a \notin \bigcup_{i \in \Omega} \alpha\left(\mathscr{C}\left(A_{i}\right)\right)$. By $\mathscr{C}\left(\bigcap_{i \in \Omega} A_{i}\right) \geq$ $\bigwedge_{i \in \Omega} \mathscr{C}\left(A_{i}\right)$, we know that

$$
\alpha\left(\mathscr{C}\left(\bigcap_{i \in \Omega} A_{i}\right)\right) \subseteq \alpha\left(\bigwedge_{i \in \Omega} \mathscr{C}\left(A_{i}\right)\right)=\bigcup_{i \in \Omega} \alpha\left(\mathscr{C}\left(A_{i}\right)\right)
$$

This shows $a \notin \alpha\left(\mathscr{C}\left(\bigcap_{i \in \Omega} A_{i}\right)\right)$. Hence, $\bigcap_{i \in \Omega} A_{i} \in \mathscr{C}^{[a]}$.

(C3) If $\left\{A_{i}: i \in \Omega\right\} \subseteq \mathscr{C}^{[a]}$ is nonempty and totally ordered by inclusion, then for each $i \in \Omega, a \notin \alpha\left(\mathscr{C}\left(A_{i}\right)\right)$. Hence, $a \notin \bigcup_{i \in \Omega} \alpha\left(\mathscr{C}\left(A_{i}\right)\right)$. By $\mathscr{C}\left(\bigcup_{i \in \Omega} A_{i}\right) \geq \bigwedge_{i \in \Omega} \mathscr{C}\left(A_{i}\right)$ we know that

$$
\alpha\left(\mathscr{C}\left(\bigcup_{i \in \Omega} A_{i}\right)\right) \subseteq \alpha\left(\bigwedge_{i \in \Omega} \mathscr{C}\left(A_{i}\right)\right)=\bigcup_{i \in \Omega} \alpha\left(\mathscr{C}\left(A_{i}\right)\right)
$$

This shows $a \notin \alpha\left(\mathscr{C}\left(\bigcup_{i \in \Omega} A_{i}\right)\right)$. Therefore, $\bigcup_{i \in \Omega} A_{i} \in \mathscr{C}^{[a]}$. The proof is completed.

Now we consider the conditions that a family of convexities forms an $M$-fuzzifying convexity. By Theorem 2, we can obtain the following result.

Corollary 23. Let $M$ be completely distributive and let $\mathscr{C}$ be an $M$-fuzzifying convexity. Then

(1) $\mathscr{C}_{[b]} \subseteq \mathscr{C}_{[a]}$ for any $a, b \in M \backslash\{\perp\}$ with $a \in \beta(b)$;

(2) $\mathscr{C}^{[b]} \subseteq \mathscr{C}^{[a]}$ for any $a, b \in \alpha(\perp)$ with $b \in \alpha(a)$.

Theorem 24. Let $M$ be completely distributive and let $\left\{\mathscr{C}^{a}\right.$ : $a \in \alpha(\perp)\}$ be a family of convexities. If $\mathscr{C}^{a}=\bigcap\left\{\mathscr{C}^{b}: a \in \alpha(b)\right\}$, for all $a \in \alpha(\perp)$, then there exists an $M$-fuzzifying convexity $\mathscr{C}$ such that $\mathscr{C}^{[a]}=\mathscr{C}^{a}$.
Proof. Suppose that $\mathscr{C}^{a}=\bigcap\left\{\mathscr{C}^{b}: a \in \alpha(b)\right\}$ for all $a \in \alpha(\perp)$. Define $\mathscr{C}: 2^{X} \rightarrow M$ by

$$
\mathscr{C}(A)=\bigwedge_{a \in \alpha(\perp)}\left(a \vee \mathscr{C}^{a}(A)\right)=\bigwedge\left\{a \in \alpha(\perp): A \notin \mathscr{C}^{a}\right\}
$$

Next we show that $\mathscr{C}^{[a]}=\mathscr{C}^{a}$ for all $a \in \alpha(\perp)$. On one hand, if $A \notin \mathscr{C}^{[a]}$, then $a \in \alpha(\mathscr{C}(A))$. There exists $b \in \alpha(\perp)$ such that $a \in \alpha(b)$ and $A \notin \mathscr{C}^{b}$. By $\mathscr{C}^{a}=\bigcap\left\{\mathscr{C}^{b}: a \in \alpha(b)\right\}$, we know that $A \notin \mathscr{C}^{b} \supseteq \mathscr{C}^{a}$. Hence, $\mathscr{C}^{[a]} \supseteq \mathscr{C}^{a}$. On the other hand, if $A \notin \mathscr{C}^{a}$, then there exists $b \in \alpha(\perp)$ such that $a \in \alpha(b)$ and $A \notin \mathscr{C}^{b}$. So $a \in \alpha(b) \subseteq \bigcup_{a \in \alpha(b)} \alpha(b)=\alpha(\bigwedge\{b \in \alpha(\perp)$ : $\left.\left.A \notin \mathscr{C}^{b}\right\}\right)=\alpha(\mathscr{C}(A))$; that is, $A \notin \mathscr{C}^{[a]}$. Then $\mathscr{C}^{a} \supseteq \mathscr{C}^{[a]}$. Therefore, $\mathscr{C}^{[a]}=\mathscr{C}^{a}$ for all $a \in \alpha(\perp)$. By Theorem 22, we can obtain that $\mathscr{C}$ is an $M$-fuzzifying convexity and $\mathscr{C}^{[a]}=\mathscr{C}^{a}$ for all $a \in \alpha(\perp)$.

Theorem 25. Let $M$ be completely distributive and let $\left\{\mathscr{C}_{a}\right.$ : $a \in M \backslash\{\perp\}\}$ be a family of convexities. If $\mathscr{C}_{a}=\bigcap\left\{\mathscr{C}_{b}: b \in\right.$ $\beta(a)\}$, for all $a \in M \backslash\{\perp\}$, then there exists an $M$-fuzzifying convexity $\mathscr{C}$ such that $\mathscr{C}_{[a]}=\mathscr{C}_{a}$.

Proof. This is straightforward.

Definition 26. Let $\mathscr{C}, \mathscr{D}$ be $M$-fuzzifying convexities on $X$. If $\mathscr{C}(A) \leq \mathscr{D}(A)$, for all $A \in 2^{X}$, which is denoted by $\mathscr{C} \leq \mathscr{D}$, then $\mathscr{C}$ is said to be coarser than $\mathscr{D}$ and $\mathscr{D}$ is said to be finer than $\mathscr{C}$.

Theorem 27. Let $\left\{\mathscr{C}_{t}: t \in T\right\}$ be a family of $M$-fuzzifying convexities on $X$. Then $\bigwedge_{t \in T} \mathscr{C}_{t}$ is an $M$-fuzzifying convexity on $X$, where $\bigwedge_{t \in T} \mathscr{C}_{t}: 2^{X} \rightarrow M$ is defined by

$$
\left(\bigwedge_{t \in T} \mathscr{C}_{t}\right)(A)=\bigwedge_{t \in T} \mathscr{C}_{t}(A)
$$

for each $A \in 2^{X}$. Obviously, $\bigwedge_{t \in T} \mathscr{C}_{t}$ is coarser than $\mathscr{C}_{t}$ for all $t \in T$.

Proof. This is straightforward.

\section{Characterizations of $M$-Fuzzifying Convex Structures by $M$-Fuzzifying Closure Operators}

In this section, we always suppose that $M$ is a completely distributive lattice with an order-reversing involution'.

Definition 28. An $M$-fuzzifying closure operator cl on $X$ is called domain finite (or algebraic) if it satisfies the following condition: for each $S \in 2^{X}$ and $x \in X$,

$(\mathrm{MDF}) \mathrm{cl}(S)(x)=\bigvee\left\{\operatorname{cl}(F)(x): F \in \mathbf{2}_{\text {fin }}^{S}\right\}$.

Theorem 29. Let $(X, \mathscr{C})$ be an $M$-fuzzifying closure structure. Then the following conditions are equivalent:

(1) $\mathscr{C}$ is an $M$-fuzzifying convexity on $X$; 
(2) the closure operator of $\mathscr{C}$ is domain finite.

(3) $\mathscr{C}$ is stable for updirected union; that is, if $\left\{A_{i}: i \in\right.$ $\Omega\} \subseteq 2^{X}$ is an updirected set, then $\mathscr{C}\left(\bigcup_{i \in \Omega} A_{i}\right) \geq$ $\bigwedge_{i \in \Omega} \mathscr{C}\left(A_{i}\right)$.

Proof. (1) $\Rightarrow$ (2) Let cl be the $M$-fuzzifying closure operator of $(X, \mathscr{C})$. By Remark 16, we know that cl satisfies (CL0)(CL3). Next we need to prove that cl satisfies (MDF). Since $(X, \mathscr{C})$ is an $M$-fuzzifying convex structure, by Theorem 22 , for each $a \in \beta(T),\left(X, \mathscr{C}^{\left[a^{\prime}\right]}\right)$ is a convex structure. Let $\mathrm{cl}_{a}$ be the closure operator of $\left(X, \mathscr{C}^{\left[a^{\prime}\right]}\right)$ for each $a \in \beta(T)$. It is obvious that $\operatorname{cl}(S)(x) \geq \bigvee\left\{\operatorname{cl}(F)(x): F \in \mathbf{2}_{\text {fin }}^{S}\right\}$. Conversely, let $a$ be any element in $\beta(T)$ with the property of $\operatorname{cl}(S)(x)>a$. Then by Theorem 12 , we have

$$
\begin{aligned}
& \operatorname{cl}(S)(x)=\bigwedge_{x \notin B \supseteq S}(\mathscr{C}(B))^{\prime}>a \\
& \Longrightarrow \forall B \supseteq S \text {, if } x \notin B \text {, then } a \prec(\mathscr{C}(B))^{\prime} \\
& \Longrightarrow \forall B \supseteq S \text {, if } x \notin B \text {, then } \mathscr{C}(B)<{ }^{\mathrm{op}} a^{\prime} \\
& \Longrightarrow \forall B \supseteq S \text {, if } x \notin B \text {, then } a^{\prime} \in \alpha(\mathscr{C}(B)) \\
& \Longrightarrow \forall B \supseteq S \text {, if } x \notin B \text {, then } B \notin \mathscr{C}^{\left[a^{\prime}\right]} \\
& \Longrightarrow x \in \mathrm{cl}_{a}(S)=\bigcup\left\{\mathrm{cl}_{a}(F): F \in \mathbf{2}_{\text {fin }}^{S}\right\} \\
& \Longrightarrow \exists F \in \mathbf{2}_{\text {fin }}^{S} \text {, s.t., } \quad x \in \mathrm{cl}_{a}(F) \\
& \Longrightarrow \exists F \in \mathbf{2}_{\text {fin }}^{\text {S }} \text {, s.t., } \forall D \supseteq F \text {, if } x \notin D \text {, } \\
& \text { then } D \notin \mathscr{C}^{\left[a^{\prime}\right]} \\
& \Longrightarrow \exists F \in \mathbf{2}_{\text {fin }}^{S} \text {, s.t., } \forall D \supseteq F \text {, if } x \notin D \text {, } \\
& \text { then } a^{\prime} \in \alpha(\mathscr{C}(D)) \\
& \Longrightarrow \exists F \in \mathbf{2}_{\text {fin }}^{S} \text {, s.t., } \forall D \supseteq F \text {, if } x \notin D \text {, } \\
& \text { then } \mathscr{C}(D) \prec{ }^{\mathrm{op}} a^{\prime} \\
& \Longrightarrow \exists F \in \mathbf{2}_{\text {fin }}^{S} \text {, s.t., } \forall D \supseteq F \text {, if } x \notin D, \\
& \text { then } a<(\mathscr{C}(D))^{\prime} \\
& \Longrightarrow \exists F \in 2_{\text {fin }}^{S} \text {, s.t., } \quad \mathrm{cl}(F)(x)=\bigwedge_{x \notin D \supseteq F}(\mathscr{C}(D))^{\prime} \geq a \\
& \Longrightarrow \bigvee\left\{\mathrm{cl}(F)(x): F \in \mathbf{2}_{\text {fin }}^{S}\right\} \geq a \text {. }
\end{aligned}
$$

By the arbitrariness of $a$ and Lemma 3, we have $\operatorname{cl}(S)(x) \leq$ $\bigvee\left\{\operatorname{cl}(F)(x): F \in \mathbf{2}_{\text {fin }}^{S}\right\}$. Therefore, $\operatorname{cl}(S)(x)=\bigvee\{\mathrm{cl}(F)(x): F \in$ $\left.2_{\text {fin }}^{S}\right\}$.

(2) $\Rightarrow$ (3) For any nonempty $\left\{A_{i}: i \in \Omega\right\} \subseteq 2^{X}$, which is an updirected set, we need to prove $\mathscr{C}\left(\bigcup_{i \in \Omega} A_{i}\right) \geq$ $\bigwedge_{i \in \Omega} \mathscr{C}\left(A_{i}\right)$.
Let $a$ be any element in $M$ with the property of $\mathscr{C}\left(\bigcup_{i \in \Omega} A_{i}\right) \geq a$. Then by Theorem 11, we have

$$
\begin{aligned}
& \mathscr{C}\left(\bigcup_{i \in \Omega} A_{i}\right) \\
& =\bigwedge_{x \notin \bigcup_{i \in \Omega} A_{i}}\left(\operatorname{cl}\left(\bigcup_{i \in \Omega} A_{i}\right)(x)\right)^{\prime} \pm a \\
& \Longrightarrow \exists x, x \notin \bigcup_{i \in \Omega} A_{i}, \quad\left(\operatorname{cl}\left(\bigcup_{i \in \Omega} A_{i}\right)(x)\right)^{\prime} \nsupseteq a \\
& \Longrightarrow \exists x, x \notin \bigcup_{i \in \Omega} A_{i}, \quad \mathrm{cl}\left(\bigcup_{i \in \Omega} A_{i}\right)(x) \npreceq a^{\prime} \\
& \Longrightarrow \exists x, x \notin \bigcup_{i \in \Omega} A_{i}, \quad \bigvee\left\{\operatorname{cl}(F)(x): F \in \mathbf{2}_{\mathrm{fin}}^{\left(\bigcup_{i \in \Omega} A_{i}\right)}\right\} \not a^{\prime} \\
& \Longrightarrow \exists x, x \notin \bigcup_{i \in \Omega} A_{i}, \quad \exists F \in \mathbf{2}_{\mathrm{fin}}^{\left(\bigcup_{i \in \Omega} A_{i}\right)}, \\
& \text { s.t., } \operatorname{cl}(F)(x) \npreceq a^{\prime} \\
& \Longrightarrow \exists x, x \notin \bigcup_{i \in \Omega} A_{i}, \quad \exists F \in \mathbf{2}_{\text {fin }}^{A_{i_{0}}}, \\
& \text { s.t., } \operatorname{cl}(F)(x) \npreceq a^{\prime} \\
& \Longrightarrow \exists x, x \notin \bigcup_{i \in \Omega} A_{i}, \quad \exists F \in \mathbf{2}_{\text {fin }}^{A_{i_{0}}}, \\
& \text { s.t., }(\mathrm{cl}(F)(x))^{\prime} \nsucceq a \text {. }
\end{aligned}
$$

Hence,

$$
\begin{aligned}
\bigwedge_{i \in \Omega} \mathscr{C}\left(A_{i}\right) & =\bigwedge_{i \in \Omega} \bigwedge_{x \notin A_{i}}\left(\operatorname{cl}\left(A_{i}\right)(x)\right)^{\prime} \\
& =\bigwedge_{i \in \Omega} \bigwedge_{x \notin A_{i}}\left(\bigvee\left\{\operatorname{cl}(F)(x): F \in \mathbf{2}_{\text {fin }}^{A_{i}}\right\}\right)^{\prime} \\
& =\bigwedge_{i \in \Omega} \bigwedge_{x \notin A_{i}} \bigwedge\left\{(\operatorname{cl}(F)(x))^{\prime}: F \in \mathbf{2}_{\text {fin }}^{A_{i}}\right\} ¥ a .
\end{aligned}
$$

Therefore, $\mathscr{C}\left(\bigcup_{i \in \Omega} A_{i}\right) \geq \bigwedge_{i \in \Omega} \mathscr{C}\left(A_{i}\right)$.

(3) $\Rightarrow$ (1) By Theorems 7 and 22, it is trivial.

By Remark 16 and Theorems 13 and 29, we can obtain the following theorem, which shows that an $M$-fuzzifying convex structure can be characterized by means of an $M$-fuzzifying closure operator, which satisfies (CL0)-(CL3) and (MDF).

Theorem 30. There is a one-to-one correspondence between $M$-fuzzifying convex structures and $M$-fuzzifying closure operators in Definition 10 satisfying (CLO) and (MDF). 


\section{4. $M$-Fuzzifying Convexity Preserving Functions}

In this section, $M$ still denotes a completely distributive lattice. We will generalize the notion of convexity preserving functions to $M$-fuzzy setting.

Theorem 31. Let $(Y, \mathscr{D})$ be an $M$-fuzzifying convex structure and $f: X \rightarrow Y$ a surjective function. Define a mapping $f^{-1}(\mathscr{D}): 2^{X} \rightarrow M$ by

$$
\forall A \in 2^{X}, \quad f^{-1}(\mathscr{D})(A)=\bigvee\left\{\mathscr{D}(B): f^{-1}(B)=A\right\}
$$

Then $\left(X, f^{-1}(\mathscr{D})\right)$ is an M-fuzzifying convex structure.

Proof. (MYC1) holds from the following equalities:

$$
\begin{gathered}
f^{-1}(\mathscr{D})(\emptyset)=\bigvee\left\{\mathscr{D}(B): f^{-1}(B)=\emptyset\right\}=\top \\
f^{-1}(\mathscr{D})(X)=\bigvee\left\{\mathscr{D}(B): f^{-1}(B)=X\right\}=\top .
\end{gathered}
$$

For (MYC2), for any nonempty set $\left\{A_{i}: i \in \Omega\right\} \subseteq$ $2^{X}$, let $a$ be any element in $M \backslash\{\perp\}$ with the property of $\bigwedge_{i \in \Omega} f^{-1}(\mathscr{D})\left(A_{i}\right)>a$. Then $\forall i \in \Omega$,

$$
\bigvee\left\{\mathscr{D}(B): f^{-1}(B)=A_{i}\right\}=f^{-1}(\mathscr{D})\left(A_{i}\right)>a .
$$

For each $i \in \Omega$, there exists $B_{i} \in 2^{Y}$ such that $f^{-1}\left(B_{i}\right)=A_{i}$ and $\mathscr{D}\left(B_{i}\right) \geq a$. Note that $f^{-1}\left(\bigcap_{i \in \Omega} B_{i}\right)=\bigcap_{i \in \Omega} f^{-1}\left(B_{i}\right)=$ $\bigcap_{i \in \Omega} A_{i}$ and $\mathscr{D}\left(\bigcap_{i \in \Omega} B_{i}\right) \geq \bigwedge_{i \in \Omega} \mathscr{D}\left(B_{i}\right) \geq a$. Finally, we have

$$
\begin{aligned}
f^{-1}(\mathscr{D})\left(\bigcap_{i \in \Omega} A_{i}\right) & =\bigvee\left\{\mathscr{D}(B): f^{-1}(B)=\bigcap_{i \in \Omega} A_{i}\right\} \\
& \geq \mathscr{D}\left(\bigcap_{i \in \Omega} B_{i}\right) \geq a .
\end{aligned}
$$

This implies that $f^{-1}(\mathscr{D})\left(\bigcap_{i \in \Omega} A_{i}\right) \geq \bigwedge_{i \in \Omega} f^{-1}(\mathscr{D})\left(A_{i}\right)$.

For (MYC3), for any nonempty set $\left\{A_{i}: i \in \Omega\right\} \subseteq 2^{X}$, which is totally ordered by inclusion, let $a$ be any element in $M \backslash\{\perp\}$ with the property of $\bigwedge_{i \in \Omega} f^{-1}(\mathscr{D})\left(A_{i}\right)>a$. Then $\forall i \in$ $\Omega, \bigvee\left\{\mathscr{D}(B): f^{-1}(B)=A_{i}\right\}=f^{-1}(\mathscr{D})\left(A_{i}\right)>a$. For each $i \in$ $\Omega$, there exists $B_{i} \in 2^{Y}$ such that $f^{-1}\left(B_{i}\right)=A_{i}$ and $\mathscr{D}\left(B_{i}\right) \geq a$. Since $f$ is surjective and $\left\{A_{i}: i \in \Omega\right\}$ is totally ordered by inclusion, we have $\left\{B_{i}: i \in \Omega\right\}$ which is totally ordered by inclusion and then $\mathscr{D}\left(\bigcup_{i \in \Omega} B_{i}\right) \geq \bigwedge_{i \in \Omega} \mathscr{D}\left(B_{i}\right) \geq a$. Note that $f^{-1}\left(\bigcup_{i \in \Omega} B_{i}\right)=\bigcup_{i \in \Omega} f^{-1}\left(B_{i}\right)=\bigcup_{i \in \Omega} A_{i}$. Finally, we have

$$
\begin{aligned}
f^{-1}(\mathscr{D})\left(\bigcup_{i \in \Omega} A_{i}\right) & =\bigvee\left\{\mathscr{D}(B): f^{-1}(B)=\bigcup_{i \in \Omega} A_{i}\right\} \\
& \geq \mathscr{D}\left(\bigcup_{i \in \Omega} B_{i}\right) \geq a .
\end{aligned}
$$

This implies that $f^{-1}(\mathscr{D})\left(\bigcup_{i \in \Omega} A_{i}\right) \geq \bigwedge_{i \in \Omega} f^{-1}(\mathscr{D})\left(A_{i}\right)$.
Definition 32. Let $(X, \mathscr{C})$ and $(Y, \mathscr{D})$ be $M$-fuzzifying convex structures. A function $f: X \rightarrow Y$ is called an $M$-fuzzifying convexity preserving function if $\mathscr{C}\left(f^{-1}(B)\right) \geq \mathscr{D}(B)$ for all $B \in 2^{Y}$.

Definition 33. Let $(X, \mathscr{C})$ and $(Y, \mathscr{D})$ be $M$-fuzzifying convex structures. A function $f: X \rightarrow Y$ is called an $M$-fuzzifying convex-to-convex function if $\mathscr{D}(f(A)) \geq \mathscr{C}(A)$ for all $A \in$ $2^{X}$.

Definition 34 . Let $(X, \mathscr{C})$ and $(Y, \mathscr{D})$ be $M$-fuzzifying convex structures. A function $f: X \rightarrow Y$ is an $M$-fuzzifying isomorphism if $f$ is a bijection, an $M$-fuzzifying convexity preserving function, and an $M$-fuzzifying convex-to-convex function.

The following theorem gives a characterization of $M$ fuzzifying convexity preserving functions.

Theorem 35. Let $(X, \mathscr{C})$ and $(Y, \mathscr{D})$ be two $M$-fuzzifying convex structures. A surjective function $f: X \rightarrow Y$ is an $M$-fuzzifying convexity preserving function if and only if $f^{-1}(\mathscr{D})(A) \leq \mathscr{C}(A)$ for all $A \in \mathbf{2}^{X}$.

Proof. Necessity. If $f: X \rightarrow Y$ is an $M$-fuzzifying convexity preserving function, then $\mathscr{C}\left(f^{-1}(B)\right) \geq \mathscr{D}(B)$ for all $B \in 2^{Y}$. Hence, for all $A \in \mathbf{2}^{X}$, we have

$$
\begin{aligned}
f^{-1}(\mathscr{D})(A) & =\bigvee\left\{\mathscr{D}(B): f^{-1}(B)=A\right\} \\
& \leq \bigvee\left\{\mathscr{C}\left(f^{-1}(B)\right): f^{-1}(B)=A\right\} \\
& =\mathscr{C}(A) .
\end{aligned}
$$

Sufficiency. If $f^{-1}(\mathscr{D})(A) \leq \mathscr{C}(A)$ for all $A \in 2^{X}$, then

$$
\begin{aligned}
\mathscr{D}(B) & \leq \bigvee\left\{\mathscr{D}(G): f^{-1}(G)=f^{-1}(B)\right\} \\
& =f^{-1}(\mathscr{D})\left(f^{-1}(B)\right) \\
& \leq \mathscr{C}\left(f^{-1}(B)\right)
\end{aligned}
$$

for all $B \in 2^{Y}$. This shows that $f: X \rightarrow Y$ is an $M$-fuzzifying convexity preserving function.

The next three theorems are trivial.

Theorem 36. If $f:(X, \mathscr{C}) \rightarrow(Y, \mathscr{D})$ and $g:(Y, \mathscr{D}) \rightarrow$ $(Z, \mathscr{H})$ are $M$-fuzzifying convexity preserving functions, then $g \circ f:(X, \mathscr{C}) \rightarrow(Z, \mathscr{H})$ is an $M$-fuzzifying convexity preserving function.

Theorem 37. Let $(X, \mathscr{C})$ and $(Y, \mathscr{D})$ be $M$-fuzzifying convex structures. Then a function $f:(X, \mathscr{C}) \rightarrow(Y, \mathscr{D})$ is an $M$-fuzzifying convexity preserving function if and only if $f$ : $\left(X, \mathscr{C}_{[a]}\right) \rightarrow\left(Y, \mathscr{D}_{[a]}\right)$ is a convexity preserving function for any $a \in M \backslash\{\perp\}$.

Theorem 38. Let $(X, \mathscr{C})$ and $(Y, \mathscr{D})$ be $M$-fuzzifying convex structures. Then a function $f:(X, \mathscr{C}) \rightarrow(Y, \mathscr{D})$ is an 
$M$-fuzzifying convexity preserving function if and only if $f$ : $\left(X, \mathscr{C}^{[a]}\right) \rightarrow\left(Y, \mathscr{D}^{[a]}\right)$ is a convexity preserving function for any $a \in \alpha(\perp)$.

\section{Quotient $M$-Fuzzifying Convex Structures}

In this section, the notions of quotient structures and quotient functions are generalized to $M$-fuzzy setting.

Theorem 39. Let $(X, \mathscr{C})$ be an $M$-fuzzifying convex structure and $f: X \rightarrow Y$ a surjective function. Define a mapping $\mathscr{D}_{f}$ : $2^{Y} \rightarrow M$ by

$$
\forall B \in \mathbf{2}^{Y}, \quad \mathscr{D}_{f}(B)=\mathscr{C}\left(f^{-1}(B)\right) .
$$

Then $\left(Y, \mathscr{D}_{f}\right)$ is an M-fuzzifying convex structure and we call $D_{f}$ a quotient $M$-fuzzifying convexity of $X$ with respect to $f$ and $\mathscr{C}$. Moreover, it is easy to see that $f$ is an M-fuzzifying convexity preserving function from $(X, \mathscr{C})$ to $\left(Y, \mathscr{D}_{f}\right)$.

Proof. (MYC1) holds from the following equalities:

$$
\begin{aligned}
& \mathscr{D}_{f}(\emptyset)=\mathscr{C}\left(f^{-1}(\emptyset)\right)=\mathscr{C}(\emptyset)=\mathrm{\top}, \\
& \mathscr{D}_{f}(Y)=\mathscr{C}\left(f^{-1}(Y)\right)=\mathscr{C}(X)=\mathrm{\top} .
\end{aligned}
$$

(MYC2) can be shown from the following fact: for any nonempty set $\left\{B_{i}: i \in \Omega\right\} \subseteq \mathbf{2}^{Y}$,

$$
\begin{aligned}
\mathscr{D}_{f}\left(\bigcap_{i \in \Omega} B_{i}\right) & =\mathscr{C}\left(f^{-1}\left(\bigcap_{i \in \Omega} B_{i}\right)\right)=\mathscr{C}\left(\bigcap_{i \in \Omega} f^{-1}\left(B_{i}\right)\right) \\
& \geq \bigwedge_{i \in \Omega} \mathscr{C}\left(f^{-1}\left(B_{i}\right)\right)=\bigwedge_{i \in \Omega} \mathscr{D}_{f}\left(B_{i}\right) .
\end{aligned}
$$

For (MYC3), if $\left\{B_{i}: i \in \Omega\right\} \subseteq \mathbf{2}^{Y}$ is nonempty and totally ordered by inclusion, then

$$
\begin{aligned}
\mathscr{D}_{f}\left(\bigcup_{i \in \Omega} B_{i}\right) & =\mathscr{C}\left(f^{-1}\left(\bigcup_{i \in \Omega} B_{i}\right)\right)=\mathscr{C}\left(\bigcup_{i \in \Omega} f^{-1}\left(B_{i}\right)\right) \\
& \geq \bigwedge_{i \in \Omega} \mathscr{C}\left(f^{-1}\left(B_{i}\right)\right)=\bigwedge_{i \in \Omega} \mathscr{D}_{f}\left(B_{i}\right) .
\end{aligned}
$$

Theorem 40. Let $(X, \mathscr{C})$ be an $M$-fuzzifying convex structure and $f: X \rightarrow Y$ a surjective function. Then $\mathscr{D}_{f}$ is the finest convexity on $Y$ such that $f$ is an $M$-fuzzifying convexity preserving function.

Proof. Let $\mathscr{D}$ be an $M$-fuzzifying convexity on $Y$ such that $f$ is an $M$-fuzzifying convexity preserving function from $(X, \mathscr{C})$ to $(Y, \mathscr{D})$; then $\forall B \in 2^{Y}, \mathscr{C}\left(f^{-1}(B)\right) \geq \mathscr{D}(B)$. We have $\forall B \in$ $2^{Y}, \mathscr{D}_{f}(B)=\mathscr{C}\left(f^{-1}(B)\right) \geq \mathscr{D}(B)$. Therefore, $\mathscr{D}_{f} \geq \mathscr{D}$.

Definition 41 . Let $(X, \mathscr{C})$ and $(Y, \mathscr{D})$ be $M$-fuzzifying convex structures. A function $f: X \rightarrow Y$ is called an $M$-fuzzifying quotient function if $f$ is surjective and $\mathscr{D}$ is a quotient $M$ fuzzifying convexity with respect to $f$ and $\mathscr{C}$.

Theorem 42. If $f:(X, \mathscr{C}) \rightarrow(Y, \mathscr{D})$ is an M-fuzzifying quotient function, then $g:(Y, \mathscr{D}) \rightarrow(Z, \mathscr{H})$ is an $M-$ fuzzifying convexity preserving function if and only if $g \circ f$ : $(X, \mathscr{C}) \rightarrow(Z, \mathscr{H})$ is an $M$-fuzzifying convexity preserving function.

Proof. Since $f:(X, \mathscr{C}) \rightarrow(Y, \mathscr{D})$ is an $M$-fuzzifying quotient function, we know that $f$ is surjective and $\forall B \in \mathbf{2}^{Y}$, $\mathscr{D}(B)=\mathscr{C}\left(f^{-1}(B)\right)$.

Necessity. Since $g:(Y, \mathscr{D}) \rightarrow(Z, \mathscr{H})$ is an $M$-fuzzifying convexity preserving function, $\forall A \in \mathbf{2}^{Z}, \mathscr{D}\left(g^{-1}(A)\right) \geq \mathscr{H}(A)$. Thus, $\forall A \in \mathbf{2}^{Z}$,

$$
\begin{aligned}
\mathscr{C}\left((g \circ f)^{-1}(A)\right) & =\mathscr{C}\left(f^{-1}\left(g^{-1}(A)\right)\right) \\
& =\mathscr{D}\left(g^{-1}(A)\right) \\
& \geq \mathscr{H}(A) .
\end{aligned}
$$

Sufficiency. Since $g \circ f:(X, \mathscr{C}) \rightarrow(Z, \mathscr{H})$ is an $M$-fuzzifying convexity preserving function, $\forall A \in \mathbf{2}^{Z}$,

$$
\begin{aligned}
\mathscr{D}\left(g^{-1}(A)\right) & =\mathscr{C}\left(f^{-1}\left(g^{-1}(A)\right)\right) \\
& =\mathscr{C}\left((g \circ f)^{-1}(A)\right) \\
& \geq \mathscr{H}(A) .
\end{aligned}
$$

Theorem 43. If $f:(X, \mathscr{C}) \rightarrow(Y, \mathscr{D})$ is a surjective $M$-fuzzifying convexity preserving function and an M-fuzzifying convex-to-convex function, then $\mathscr{D}$ is a quotient M-fuzzifying convexity. Moreover, $f$ is an M-fuzzifying quotient function.

Proof. Since $f:(X, \mathscr{C}) \rightarrow(Y, \mathscr{D})$ is a surjective $M$-fuzzifying convexity preserving function and an $M$-fuzzifying convex-to-convex function, we have $\forall B \in \mathbf{2}^{Y}, \mathscr{C}\left(f^{-1}(B)\right) \geq$ $\mathscr{D}(B)$ and $\forall A \in 2^{X}, \mathscr{D}(f(A)) \geq \mathscr{C}(A)$. Since $f$ is surjective, for all $B \in \mathbf{2}^{Y}, f\left(f^{-1}(B)\right)=B$. Hence,

$$
\mathscr{D}(B)=\mathscr{D}\left(f\left(f^{-1}(B)\right)\right) \geq \mathscr{C}\left(f^{-1}(B)\right) \geq \mathscr{D}(B) .
$$

So $\mathscr{C}\left(f^{-1}(B)\right)=\mathscr{D}(B)$ for each $B \in \mathbf{2}^{Y}$ and then $\mathscr{D}$ is a quotient $M$-fuzzifying convexity.

Based on Theorem 39, we can obtain the following result.

Theorem 44. Let $(X, \mathscr{C})$ be an $M$-fuzzifying convex structure and let $R$ be an equivalence relation defined on $X$. Let $X / R$ be the usual quotient set and let $\pi$ be the projection mapping from $X$ to $X / R$. Define $\mathscr{D}: \mathbf{2}^{(X / R)} \rightarrow M$ by

$$
\forall B \in \mathbf{2}^{(X / R)}, \quad \mathscr{D}(B)=\mathscr{C}\left(\pi^{-1}(B)\right) .
$$

Then $\mathscr{D}$ is an $M$-fuzzifying convexity on $X / R$ and $(X / R, \mathscr{D})$ is a quotient $M$-fuzzifying convex structure of $(X, \mathscr{C})$. 


\section{Substructures and Disjoint Sums of $M$-Fuzzifying Convex Structures}

In this section, $M$ still denotes a completely distributive lattice. We will give the substructures and disjoint sums of $M$-fuzzifying convex structures and discuss some of their fundamental properties.

Theorem 45. Let $(X, \mathscr{C})$ be an $M$-fuzzifying convex structure, $\emptyset \neq Y \subseteq X$. Then $(Y, \mathscr{C} \mid Y)$ is an $M$-fuzzifying convex structure on $Y$, where $\forall A \in \mathbf{2}^{Y},(\mathscr{C} \mid Y)(A)=\bigvee\{\mathscr{C}(B)$ : $\left.B \in \mathbf{2}^{X}, B \cap Y=A\right\}$. One calls $(Y, \mathscr{C} \mid Y)$ an $M$-fuzzifying substructure of $(X, \mathscr{C})$.

Proof. (1) Clearly, $(\mathscr{C} \mid Y)(\emptyset)=(\mathscr{C} \mid Y)(Y)=\mathrm{T}$.

(2) For any $\left\{A_{i}: i \in \Omega\right\} \subseteq \mathbf{2}^{Y}$, we have

$$
\begin{aligned}
\bigwedge_{i \in \Omega}(\mathscr{C} \mid Y)\left(A_{i}\right) & =\bigwedge_{i \in \Omega} \bigvee\left\{\mathscr{C}(B): B \in \mathbf{2}^{X}, B \cap Y=A_{i}\right\} \\
& =\bigvee_{f \in \Pi_{i \in \Omega} H_{i}} \bigwedge_{i \in \Omega} \mathscr{C}(f(i)) \\
& \leq \bigvee_{f \in \Pi_{i \in \Omega} H_{i}} \mathscr{C}\left(\bigcap_{i \in \Omega} f(i)\right),
\end{aligned}
$$

where $H_{i}=\left\{B: B \in \mathbf{2}^{X}, B \cap Y=A_{i}\right\}(i \in \Omega)$. Since $\left(\bigcap_{i \in \Omega} f(i)\right) \cap Y=\bigcap_{i \in \Omega}(f(i) \cap Y)=\bigcap_{i \in \Omega} A_{i}$, we have $(\mathscr{C} \mid Y)\left(\bigcap_{i \in \Omega} A_{i}\right) \geq \bigwedge_{i \in \Omega}(\mathscr{C} \mid Y)\left(A_{i}\right)$.

(3) For any $\left\{A_{i}: i \in \Omega\right\} \subseteq 2^{Y} \subseteq 2^{X}$, which is nonempty and totally ordered by inclusion, let $a$ be any element in $M \backslash\{\perp\}$ with the property of $\bigwedge_{i \in \Omega}(\mathscr{C} \mid Y)\left(A_{i}\right)>a$; that is, $\bigwedge_{i \in \Omega} \bigvee\left\{\mathscr{C}(B): B \in \mathbf{2}^{X}, B \cap Y=A_{i}\right\}>a$. Then for each $i \in \Omega$, there exists $B_{i} \in 2^{X}$ such that $B_{i} \cap Y=A_{i}$ and $\mathscr{C}\left(B_{i}\right) \geq a$; that is, $B_{i} \in \mathscr{C}_{[a]}$. By Theorem 21, for each $a \in M \backslash\{\perp\},\left(X, \mathscr{C}_{[a]}\right)$ is a convex structure. Let $\mathrm{co}_{a}$ denote the hull operator of $\left(X, \mathscr{C}_{[a]}\right)$ for each $a \in M \backslash\{\perp\}$. Then $\operatorname{co}_{a}\left(A_{i}\right) \in \mathscr{C}_{[a]}$ for all $i \in \Omega$. Since $\left\{A_{i}: i \in \Omega\right\} \subseteq 2^{Y}$ is nonempty and totally ordered by inclusion, $\left\{\operatorname{co}_{a}\left(A_{i}\right): i \in \Omega\right\}$ is nonempty and totally ordered by inclusion. Hence, $\bigcup_{i \in \Omega} \operatorname{co}_{a}\left(A_{i}\right) \in \mathscr{C}_{[a]}$; that is, $\mathscr{C}\left(\bigcup_{i \in \Omega} \operatorname{co}_{a}\left(A_{i}\right)\right) \geq a$. By Theorem 5, $\left(\bigcup_{i \in \Omega} \operatorname{co}_{a}\left(A_{i}\right)\right) \cap Y=$ $\left(\bigcup_{i \in \Omega} \operatorname{co}_{a}\left(B_{i} \cap Y\right)\right) \cap Y=\bigcup_{i \in \Omega} \operatorname{co}_{a}\left(B_{i} \cap Y\right) \cap Y=\bigcup_{i \in \Omega}\left(B_{i} \cap Y\right)=$ $\bigcup_{i \in \Omega} A_{i}$. So we have $(\mathscr{C} \mid Y)\left(\bigcup_{i \in \Omega} A_{i}\right) \geq a$. This implies that $(\mathscr{C} \mid Y)\left(\bigcup_{i \in \Omega} A_{i}\right) \geq \bigwedge_{i \in \Omega}(\mathscr{C} \mid Y)\left(A_{i}\right)$.

Theorem 46. Let $\left\{\left(X_{t}, \mathscr{C}_{t}\right)\right\}_{t \in T}$ be a family of pairwise disjoint $M$-fuzzifying convex structures; that is, $X_{t_{1}} \cap X_{t_{2}}=\emptyset$ for $t_{1} \neq$ $t_{2}$. Consider the set $X=\bigcup_{t \in T} X_{t}$ and $\forall t \in T, j_{t}: X_{t} \rightarrow X$ is the usual inclusion mapping (i.e., $\forall x \in X_{t}, j_{t}(x)=x$ ). Define a mapping $\mathscr{C}: 2^{X} \rightarrow M$ by $\forall A \in 2^{X}, \mathscr{C}(A)=\bigwedge_{t \in T} \mathscr{C}_{t}\left(j_{t}^{-1}(A)\right)$. Then $\mathscr{C}$ is an $M$-fuzzifying convexity on $X . \mathscr{C}$ is called an $M-$ fuzzifying sum convexity of $\left\{\mathscr{C}_{t}\right\}_{t \in T}$ and is denoted by $\sum_{t \in T} \mathscr{C}_{t}$. The $M$-fuzzifying convex structure $\left(X, \sum_{t \in T} \mathscr{C}_{t}\right)$ is called the $M$-fuzzifying sum convex structure of $\left\{\left(X_{t}, \mathscr{C}_{t}\right)\right\}_{t \in T}$, written as $\sum_{t \in T}\left(X_{t}, \mathscr{C}_{t}\right)$.

Proof. (1) Clearly, $\mathscr{C}(\emptyset)=\mathscr{C}(X)=\mathrm{T}$.
(2) For any nonempty set $\left\{A_{i}: i \in \Omega\right\} \subseteq 2^{X}$, we have

$$
\begin{aligned}
\bigwedge_{i \in \Omega} \mathscr{C}\left(A_{i}\right) & =\bigwedge_{i \in \Omega} \bigwedge_{t \in T} \mathscr{C}_{t}\left(j_{t}^{-1}\left(A_{i}\right)\right) \\
& =\bigwedge_{t \in T} \bigwedge_{i \in \Omega} \mathscr{C}_{t}\left(j_{t}^{-1}\left(A_{i}\right)\right) \\
& \leq \bigwedge_{t \in T} \mathscr{C}_{t}\left(\bigcap_{i \in \Omega} j_{t}^{-1}\left(A_{i}\right)\right) \\
& =\bigwedge_{t \in T} \mathscr{C}_{t}\left(j_{t}^{-1}\left(\bigcap_{i \in \Omega} A_{i}\right)\right) \\
& =\mathscr{C}\left(\bigcap_{i \in \Omega} A_{i}\right) .
\end{aligned}
$$

(3) For any $\left\{A_{i}: i \in \Omega\right\} \subseteq \mathbf{2}^{X}$, which is nonempty and totally ordered by inclusion, we have

$$
\begin{aligned}
\bigwedge_{i \in \Omega} \mathscr{C}\left(A_{i}\right) & =\bigwedge_{i \in \Omega} \bigwedge_{t \in T} \mathscr{C}_{t}\left(j_{t}^{-1}\left(A_{i}\right)\right) \\
& =\bigwedge_{t \in T} \bigwedge_{i \in \Omega} \mathscr{C}_{t}\left(j_{t}^{-1}\left(A_{i}\right)\right) \\
& \leq \bigwedge_{t \in T} \mathscr{C}_{t}\left(\bigcup_{i \in \Omega} j_{t}^{-1}\left(A_{i}\right)\right) \\
& =\bigwedge_{t \in T} \mathscr{C}_{t}\left(j_{t}^{-1}\left(\bigcup_{i \in \Omega} A_{i}\right)\right) \\
& =\mathscr{C}\left(\bigcup_{i \in \Omega} A_{i}\right) .
\end{aligned}
$$

Remark 47. Let $\left\{\left(X_{t}, \mathscr{C}_{t}\right)\right\}_{t \in T}$ be a family of $M$-fuzzifying convex structures. Consider $\forall t \in T, Y_{t}=X_{t} \times\{t\}$. Then $Y_{t} \cap Y_{s}=\emptyset$ for $t \neq s$. For each $t \in T$, the usual mapping $p_{t}: Y_{t} \rightarrow X_{t},(x, t) \mapsto x$ is one-to-one and onto and it naturally induces the mapping from $2^{Y_{t}}$ to $2^{X_{t}}$, still denoted by $p_{t}$. Define $\mathscr{D}_{t}: \mathbf{2}^{Y_{t}} \rightarrow M$ such that $\mathscr{D}_{t}(A)=\mathscr{C}_{t}\left(p_{t}(A)\right)$ for each $A \in 2^{Y_{t}}$. Then one can easily verify that it is an $M$-fuzzifying convexity on $Y_{t}$ and $p_{t}:\left(Y_{t}, \mathscr{D}_{t}\right) \rightarrow\left(X_{t}, \mathscr{C}_{t}\right)$ is an $M$-fuzzifying isomorphism. Thus, we may identify $\sum_{t \in T}\left(X_{t}, \mathscr{C}_{t}\right)$ with $\sum_{t \in T}\left(Y_{t}, \mathscr{D}_{t}\right)$. In the sequel, we will assume that any family of $M$-fuzzifying convex structures has an $M$-fuzzifying sum convex structure (up to an $M$-fuzzifying isomorphism), but in the proof we still tacitly assume that the discussed family consists of pairwise disjoint $M$-fuzzifying convex structures. This is because there is no difference between two families of $M$-fuzzifying convex structures from a point of isomorphism.

Theorem 48. Let $(X, \mathscr{C})=\sum_{t \in T}\left(X_{t}, \mathscr{C}_{t}\right)$. Then $\mathscr{C}$ is the finest $M$-fuzzifying convexity on $X$ such that $\left\{j_{t}: t \in T\right\}$ are $M$ fuzzifying convexity preserving functions.

Proof. If there is an $M$-fuzzifying convexity $\mathscr{D}$ on $X$ such that $\forall t \in T, j_{t}: X_{t} \rightarrow X$ is an $M$-fuzzifying convexity 
preserving function, then for each $t \in T$ and $A \in \mathbf{2}^{X}, \mathscr{D}(A) \leq$ $\mathscr{C}_{t}\left(\left(j_{t}\right)^{-1}(A)\right)$ and thus $\mathscr{D}(A) \leq \bigwedge_{t \in T} \mathscr{C}_{t}\left(\left(j_{t}\right)^{-1}(A)\right)=\mathscr{C}(A)$. Therefore, $\mathscr{D} \leq \mathscr{C}$.

Theorem 49. Let $(X, \mathscr{C})=\sum_{t \in T}\left(X_{t}, \mathscr{C}_{t}\right)$ and let $(Y, \mathscr{D})$ be an $M$-fuzzifying convex structure. Then $f$ is an M-fuzzifying convexity preserving function from $(X, \mathscr{C})$ to $(Y, \mathscr{D})$ if and only if the composition $f \circ j_{t}$ is an $M$-fuzzifying convexity preserving function for each $t \in T$.

Proof. Necessity. It is easy to obtain the necessity by Theorem 42.

Sufficiency. Since the composition $f \circ j_{t}$ is an $M$-fuzzifying convexity preserving function for each $t \in T$, we have for each $A \in \mathbf{2}^{Y}, \mathscr{D}(A) \leq \mathscr{C}_{t}\left(\left(f \circ j_{t}\right)^{-1}(A)\right)=\mathscr{C}_{t}\left(\left(j_{t}\right)^{-1}\left(f^{-1}(A)\right)\right)$. And thus $\mathscr{D}(A) \leq \bigwedge_{t \in T} \mathscr{C}_{t}\left(\left(j_{t}\right)^{-1}\left(f^{-1}(A)\right)\right)=\mathscr{C}\left(f^{-1}(A)\right)$. It implies that $f$ is an $M$-fuzzifying convexity preserving function from $(X, \mathscr{C})$ to $(Y, \mathscr{D})$.

Theorem 50. Let $(X, \mathscr{C})=\sum_{t \in T}\left(X_{t}, \mathscr{C}_{t}\right)$ and $Y_{t} \subseteq X_{t}$ for each $t \in T$. Then $\mathscr{C} \mid Y=\sum_{t \in T}\left(\mathscr{C}_{t} \mid Y_{t}\right)$, where $Y=\bigcup_{t \in T} Y_{t}$.

Proof. Let $j_{t}: X_{t} \rightarrow X(\forall t \in T)$ be the usual inclusion mapping and $j_{t} \mid Y_{t}: Y_{t} \rightarrow Y(\forall t \in T)$ be the restriction of $j_{t}$ to $Y_{t}$. Then by Theorems 45 and 46 , we obtain, $\forall B \in \mathbf{2}^{Y}$,

$$
\begin{aligned}
\sum_{t \in T}\left(\mathscr{C}_{t} \mid Y_{t}\right)(B) \\
=\bigwedge_{t \in T}\left(\mathscr{C}_{t} \mid Y_{t}\right)\left(B \cap Y_{t}\right) \\
=\bigwedge_{t \in T}\left\{\bigvee \mathscr{C}_{t}(D): D \in \mathbf{2}^{X_{t}}, D \cap Y_{t}=B \cap Y_{t}\right\} .
\end{aligned}
$$

We also have $\forall B \in \mathbf{2}^{Y}$,

$$
\begin{aligned}
(\mathscr{C} \mid Y)(B) & =\bigvee\left\{\mathscr{C}(D): D \in \mathbf{2}^{X}, D \cap Y=B\right\} \\
& =\bigvee\left\{\bigwedge_{t \in T} \mathscr{C}_{t}\left(j_{t}^{-1}(D)\right): D \in \mathbf{2}^{X}, D \cap Y=B\right\} \\
& =\bigvee\left\{\bigwedge_{t \in T} \mathscr{C}_{t}\left(D \cap X_{t}\right): D \in \mathbf{2}^{X}, D \cap Y=B\right\} .
\end{aligned}
$$

Note that for any $D \in 2^{X}$ with $D \cap Y=B$, we know $\left(D \cap X_{t}\right) \cap$ $Y_{t}=D \cap Y_{t}=B \cap Y_{t}$. Hence, $\forall t \in T$,

$$
\mathscr{C}_{t}\left(D \cap X_{t}\right) \leq \bigvee\left\{\mathscr{C}_{t}(D): D \in \mathbf{2}^{X_{t}}, D \cap Y_{t}=B \cap Y_{t}\right\} .
$$

Therefore,

$$
\begin{aligned}
(\mathscr{C} \mid Y)(B) & =\bigvee\left\{\bigwedge_{t \in T} \mathscr{C}_{t}\left(D \cap X_{t}\right): D \in \mathbf{2}^{X}, D \cap Y=B\right\} \\
& \leq \bigwedge_{t \in T} \bigvee\left\{\mathscr{C}_{t}(D): D \in \mathbf{2}^{X_{t}}, D \cap Y_{t}=B \cap Y_{t}\right\} \\
& =\sum_{t \in T}\left(\mathscr{C}_{t} \mid Y_{t}\right)(B)
\end{aligned}
$$

for every $B \in \mathbf{2}^{Y}$. Conversely, suppose that $a \prec \sum_{t \in T}\left(\mathscr{C}_{t} \mid\right.$ $\left.Y_{t}\right)(B)=\bigwedge_{t \in T} \bigvee\left\{\mathscr{C}_{t}(D): D \in \mathbf{2}^{X_{t}}, D \cap Y_{t}=B \cap Y_{t}\right\}$ for $B \in \mathbf{2}^{Y}$. For any $t \in T$, there exists $D_{t} \in \mathbf{2}^{X_{t}}$ such that $D_{t} \cap$ $Y_{t}=B \cap Y_{t}$ and $\mathscr{C}_{t}\left(D_{t}\right) \geq a$. Let $D=\bigcup_{t \in T} D_{t} \in \mathbf{2}^{X}$. Then $D \cap Y=D \cap \bigcup_{t \in T} Y_{t}=\bigcup_{t \in T}\left(D \cap Y_{t}\right)=\bigcup_{t \in T} B \cap Y_{t}=B$ (where $\left.B \in \mathbf{2}^{Y}\right)$, and $\forall t \in T, \mathscr{C}_{t}\left(D \cap X_{t}\right)=\mathscr{C}_{t}\left(D_{t}\right) \geq a$. Hence, $a \leq \bigwedge_{t \in T} \mathscr{C}_{t}\left(D \cap X_{t}\right)$, where $D \cap Y=B$. We obtain $(\mathscr{C} \mid Y)(B)=\bigvee\left\{\bigwedge_{t \in T} \mathscr{C}_{t}\left(D \cap X_{t}\right): D \in \mathbf{2}^{X}, D \cap Y=B\right\} \geq a$. So $(\mathscr{C} \mid Y)(B) \geq \sum_{t \in T}\left(\mathscr{C}_{t} \mid Y_{t}\right)(B)$ for all $B \in \mathbf{2}^{Y}$. Finally, we have shown that $\mathscr{C} \mid Y=\sum_{t \in T}\left(\mathscr{C}_{t} \mid Y_{t}\right)$.

\section{Products and Joins of $M$-Fuzzifying Convex Structures}

In this section, we give subbases and bases of $M$-fuzzifying convex structures and then products and joins of $M$ fuzzifying convex structures can be defined. $\bigcup_{\lambda \in \Lambda}^{\mathrm{dir}} B_{\lambda}$ denotes the union of an updirected set $\left\{B_{\lambda}: \lambda \in \Lambda\right\} \subseteq \mathbf{2}^{X}$ and $M$ is a completely distributive lattice in this section.

By Theorem 27, we can give the following definition.

Definition 51. Let $\varphi: \mathbf{2}^{X} \rightarrow M$ be a mapping. The $M$ fuzzifying convex structure $(X, \mathscr{C})$ generated by $\varphi$ is given by

$$
\forall A \in \mathbf{2}^{X}, \quad \mathscr{C}(A)=\bigwedge\{\mathscr{D}(A): \varphi \leq \mathscr{D} \in \mathfrak{H}\},
$$

where $\mathfrak{H}$ denotes all the $M$-fuzzifying convexities on $X$. Then $\varphi$ is called a subbase of the $M$-fuzzifying convexity $\mathscr{C}$. Alternatively, we say that $\varphi$ generates the convexity $\mathscr{C}$.

Definition 52 . Let $(X, \mathscr{C})$ be an $M$-fuzzifying convex structure and $\mathscr{B}: 2^{X} \rightarrow M$ a mapping with $\mathscr{B} \leq \mathscr{C} . \mathscr{B}$ is called a base of $(X, \mathscr{C})$ if it satisfies the following condition:

$$
\forall A \in \mathbf{2}^{X}, \quad \mathscr{C}(A)=\bigvee_{\bigcup_{\lambda \in \Lambda}^{\mathrm{dir}} B_{\lambda}=A} \bigwedge_{\lambda \in \Lambda} \mathscr{B}\left(B_{\lambda}\right) .
$$

Theorem 53. Let $(X, \mathscr{C})$ be an $M$-fuzzifying convex structure. If $\mathscr{B}$ is a base of $(X, \mathscr{C})$, then $\mathscr{B}$ is a subbase of $(X, \mathscr{C})$.

Proof. Let $(X, \mathscr{D})$ be an $M$-fuzzifying convex structure and $\mathscr{B} \leq \mathscr{D}$. By the definition of bases, we have $\forall A \in \mathbf{2}^{X}$,

$$
\begin{aligned}
\mathscr{C}(A) & =\bigvee_{\bigcup_{\lambda \in \Lambda}^{\mathrm{dir}} B_{\lambda}=A} \bigwedge_{\lambda \in \Lambda} \mathscr{B}\left(B_{\lambda}\right) \\
& \leq \bigvee_{\bigcup_{\lambda \in \Lambda}^{\mathrm{dir}} B_{\lambda}=A} \bigwedge_{\lambda \in \Lambda} \mathscr{D}\left(B_{\lambda}\right) \\
& \leq \bigvee_{\bigcup_{\lambda \in \Lambda}^{\mathrm{dir}} B_{\lambda}=A} \mathscr{D}\left(\bigcup_{\lambda \in \Lambda} B_{\lambda}\right) \\
& =\mathscr{D}(A) .
\end{aligned}
$$


Theorem 54. Let $(X, \mathscr{B})$ be an $M$-fuzzifying closure structure. Define a mapping $\mathscr{C}: 2^{X} \rightarrow M$ by

$$
\forall A \in 2^{X}, \quad \mathscr{C}(A)=\bigvee_{\bigcup_{\lambda \in \Lambda}^{\mathrm{dir}} B_{\lambda}=A} \bigwedge_{\lambda \in \Lambda} \mathscr{B}\left(B_{\lambda}\right) .
$$

Then $\mathscr{C}$ is an $M$-fuzzifying convexity with $\mathscr{B}$ as its base.

Proof. Obviously, (MYC1) and (MYC3) hold. Next we prove $\mathscr{C}\left(\bigcap_{i \in \Omega} A_{i}\right) \geq \bigwedge_{i \in \Omega} \mathscr{C}\left(A_{i}\right)$ for any nonempty $\left\{A_{i}: i \in \Omega\right\} \subseteq$ $2^{X}$; that is,

$$
\bigvee_{\bigcup_{\lambda \in \Lambda}^{\mathrm{dir}} B_{\lambda}=\bigwedge_{i \in \Omega} A_{i}} \bigwedge_{\lambda \in \Lambda} \mathscr{B}\left(B_{\lambda}\right) \geq \bigwedge_{i \in \Omega} \bigvee_{\bigcup_{j \in J_{i}}^{\mathrm{dir}} G_{i, j}=A_{i}} \bigwedge_{j \in J_{i}} \mathscr{B}\left(G_{i, j}\right)
$$

for any nonempty $\left\{A_{i}: i \in \Omega\right\} \subseteq 2^{X}$. Let $a$ be any element in $M$ with the property of

$$
a \prec \bigwedge_{i \in \Omega} \bigvee_{\bigcup_{j \in J_{i}}^{\mathrm{dir}} G_{i, j}=A_{i}} \bigwedge_{j \in J_{i}} \mathscr{B}\left(G_{i, j}\right) .
$$

Then for each $i \in \Omega$, there exists a updirected set $\left\{G_{i, j}: j \in\right.$ $\left.J_{i}\right\} \subseteq 2^{X}$ such that $\bigcup\left\{G_{i, j}: j \in J_{i}\right\}=A_{i}$ and $\forall j \in J_{i}, \mathscr{B}\left(G_{i, j}\right) \geq$ a. Thus,

$$
\bigcap_{i \in \Omega} A_{i}=\bigcap_{i \in \Omega} \bigcup\left\{G_{i, j}: j \in J_{i}\right\}=\bigcup\left\{\bigcap_{i \in \Omega} G_{i, f(i)}: f \in \prod_{i \in \Omega} J_{i}\right\}
$$

and $\mathscr{B}\left(G_{i, f(i)}\right) \geq a$. We have $\bigvee_{\bigcup_{\lambda \in \Lambda}^{\text {dir }} B_{\lambda}=\bigcap_{i \in \Omega} A_{i}} \bigwedge_{\lambda \in \Lambda} \mathscr{B}\left(B_{\lambda}\right) \geq a$. This implies that $\mathscr{C}\left(\bigcap_{i \in \Omega} A_{i}\right) \geq \bigwedge_{i \in \Omega} \mathscr{C}\left(A_{i}\right)$. By the definition of $\mathscr{C}$, we know that $\mathscr{B}$ is a base of $(X, \mathscr{C})$.

Theorem 55. Let $(X, \mathscr{C})$ be an $M$-fuzzifying convex structure with $\varphi$ as its subbase, where $\varphi(\emptyset)=\varphi(X)=\top$. Define $a$ mapping $\mathscr{B}: 2^{X} \rightarrow M$ by

$$
\forall B \in 2^{X}, \quad \mathscr{B}(B)=\bigvee_{\bigcap_{\lambda \in \Lambda} B_{\lambda}=B} \bigwedge_{\lambda \in \Lambda} \varphi\left(B_{\lambda}\right) .
$$

Then $\mathscr{B}$ is a base of $(X, \mathscr{C})$.

Proof. First, we prove that $\mathscr{B}$ satisfies (MYC1) and (MYC2). Obviously, (MYC1) holds.

For any nonempty $\left\{B_{i}: i \in \Omega\right\} \subseteq \mathbf{2}^{X}$, let $a$ be any element in $M$ with the property of

$$
a \prec \bigwedge_{i \in \Omega} \bigvee_{j \in J_{i}} G_{i, j}=\bigwedge_{i} \varphi\left(G_{i, j}\right)=\bigwedge_{i \in \Omega} \mathscr{B}\left(B_{i}\right)
$$

Then for each $i \in \Omega$, there exists a set $\left\{G_{i, j}: j \in J_{i}\right\} \subseteq 2^{X}$ such that $\bigcap\left\{G_{i, j}: j \in J_{i}\right\}=B_{i}$ and $\forall j \in J_{i}, \varphi\left(G_{i, j}\right) \geq a$. Thus, $\bigcap_{i \in \Omega} B_{i}=\bigcap_{i \in \Omega} \bigcap\left\{G_{i, j}: j \in J_{i}\right\}$ and $\varphi\left(G_{i, j}\right) \geq a$. We have $\mathscr{B}\left(\bigcap_{i \in \Omega} B_{i}\right)=\bigvee_{\bigcap_{\lambda \in \Lambda} B_{\lambda}=\bigcap_{i \in \Omega} B_{i}} \bigwedge_{\lambda \in \Lambda} \varphi\left(B_{\lambda}\right) \geq a$. This implies that $\mathscr{B}\left(\bigcap_{i \in \Omega} B_{i}\right) \geq \bigwedge_{i \in \Omega} \mathscr{B}\left(B_{i}\right)$ and then (MYC2) holds. So $(X, \mathscr{B})$ is an $M$-fuzzifying closure structure. By Theorem 54 , there exists an $M$-fuzzifying convex structure $(X, \mathscr{D})$ with $\mathscr{B}$ as its base.
Next we need to prove that $\mathscr{C}=\mathscr{D}$. Obviously, $\mathscr{C} \leq \mathscr{D}$. On the other hand, $\forall A \in 2^{X}$,

$$
\begin{aligned}
& \mathscr{D}(A)=\bigvee_{\bigcup_{\lambda \in \Lambda}^{\mathrm{dir}} B_{\lambda}=A} \bigwedge_{\lambda \in \Lambda} \mathscr{B}\left(B_{\lambda}\right) \\
& =\bigvee_{\bigcup_{\lambda \in \Lambda}^{\mathrm{dir}} B_{\lambda}=A} \bigwedge_{\lambda \in \Lambda} \bigvee_{\bigcap_{\beta \in \Delta_{\lambda}} G_{\beta}=B_{\lambda}} \bigwedge_{\beta \in \Delta_{\lambda}} \varphi\left(G_{\beta}\right) \\
& \leq \bigvee_{\bigcup_{\lambda \in \Lambda}^{\mathrm{dir}} B_{\lambda}=A} \bigwedge_{\lambda \in \Lambda} \bigvee_{\bigcap_{\beta \in \Delta_{\lambda}} G_{\beta}=B_{\lambda}} \bigwedge_{\beta \in \Delta_{\lambda}} \mathscr{C}\left(G_{\beta}\right) \\
& \leq \bigvee_{\bigcup_{\lambda \in \Lambda}^{\mathrm{dir}} B_{\lambda}=A} \bigwedge_{\lambda \in \Lambda} \bigvee_{\bigcap_{\beta \in \Delta_{\lambda}} G_{\beta}=B_{\lambda}} \mathscr{C}\left(\bigcap_{\beta \in \Delta_{\lambda}} G_{\beta}\right) \\
& =\bigvee_{\bigcup_{\lambda \in \Lambda}^{\mathrm{dir}} B_{\lambda}=A} \bigwedge_{\lambda \in \Lambda} \mathscr{C}\left(B_{\lambda}\right) \\
& \leq \bigvee_{\bigcup_{\lambda \in \Lambda}^{\mathrm{dir}} B_{\lambda}=A} \mathscr{C}\left(\bigcup_{\lambda \in \Lambda}^{\mathrm{dir}} B_{\lambda}\right) \\
& =\mathscr{C}(A) \text {. }
\end{aligned}
$$

Thus, $\mathscr{D}=\mathscr{C}$. Therefore, $\mathscr{B}$ is a base of $(X, \mathscr{C})$.

Theorem 56. Let $(X, \mathscr{C})$ and $(Y, \mathscr{D})$ be $M$-fuzzifying convex structures, let $f:(X, \mathscr{C}) \rightarrow(Y, \mathscr{D})$ be a function, and let $\varphi$ be a subbase of $(Y, \mathscr{D})$, where $\varphi(\emptyset)=\varphi(X)=\top$. Then $f$ is an $M$ fuzzifying convexity preserving function if and only if $\forall B \in \mathbf{2}^{Y}$, $\mathscr{C}\left(f^{-1}(B)\right) \geq \varphi(B)$.

Proof. Necessity. Since $\mathscr{D}(B) \geq \varphi(B)$ for each $B \in 2^{Y}$, we can obtain that the necessity holds.

Sufficiency. Consider $\forall B \in \mathbf{2}^{Y}$,

$$
\begin{aligned}
& \mathscr{D}(B)=\bigvee_{\bigcup_{\lambda \in \Lambda}^{\mathrm{dir}} B_{\lambda}=B} \bigwedge_{\lambda \in \Lambda} \mathscr{B}\left(B_{\lambda}\right) \\
& =\bigvee_{\bigcup_{\lambda \in \Lambda}^{\mathrm{dir}} B_{\lambda}=B} \bigwedge_{\lambda \in \Lambda} \bigvee_{\bigcap_{\beta \in \Delta_{\lambda}} G_{\beta}=B_{\lambda}} \bigwedge_{\beta \in \Delta_{\lambda}} \varphi\left(G_{\beta}\right) \\
& \leq \bigvee_{\bigcup_{\lambda \in \Lambda}^{\mathrm{dir}} B_{\lambda}=B} \bigwedge_{\lambda \in \Lambda} \bigvee_{\bigcap_{\beta \in \Delta_{\lambda}} G_{\beta}=B_{\lambda}} \bigwedge_{\beta \in \Delta_{\lambda}} \mathscr{C}\left(f^{-1}\left(G_{\beta}\right)\right) \\
& \leq \bigvee_{\bigcup_{\lambda \in \Lambda}^{\mathrm{dir}} B_{\lambda}=B} \bigwedge_{\lambda \in \Lambda} \bigvee_{\bigcap_{\beta \in \Delta_{\lambda}} G_{\beta}=B_{\lambda}} \mathscr{C}\left(f^{-1}\left(\bigcap_{\beta \in \Delta_{\lambda}} G_{\beta}\right)\right) \\
& =\bigvee_{\bigcup_{\lambda \in \Lambda}^{\mathrm{dir}} B_{\lambda}=B} \bigwedge_{\lambda \in \Lambda} \mathscr{C}\left(f^{-1}\left(B_{\lambda}\right)\right) \\
& \leq \bigvee_{\bigcup_{\lambda \in \Lambda}^{\mathrm{dir}} B_{\lambda}=B} \mathscr{C}\left(f^{-1}\left(\bigcup_{\lambda \in \Lambda}^{\operatorname{dir}} B_{\lambda}\right)\right) \\
& =\mathscr{C}\left(f^{-1}(B)\right) \text {. }
\end{aligned}
$$


Based on Definition 51, we can define the product of $M$ fuzzifying convex structures as follows.

Definition 57. Let $\left\{\left(X_{t}, \mathscr{C}_{t}\right)\right\}_{t \in T}$ be a family of $M$-fuzzifying convex structures. Let $X$ be the product of the sets of $X_{t}$ for $t \in T$ and let $\pi_{t}: X \rightarrow X_{t}$ denote the projection for each $t \in T$. Define a mapping $\varphi: 2^{X} \rightarrow M$ by $\varphi(A)=$ $\bigvee_{t \in T} \bigvee_{\left(\pi_{t}\right)^{-1}(B)=A} \mathscr{C}_{t}(B)$ for each $A \in \mathbf{2}^{X}$. Then the product convexity $\mathscr{C}$ of $X$ is the one generated by the subbase $\varphi$. The resulting $M$-fuzzifying convex structure $(X, \mathscr{C})$ is called the product of $\left\{\left(X_{t}, \mathscr{C}_{t}\right)\right\}_{t \in T}$ and is denoted by $\prod_{t \in T}\left(X_{t}, \mathscr{C}_{t}\right)$.

Theorem 58. Let $(X, \mathscr{C})$ be the product of $\left\{\left(X_{t}, \mathscr{C}_{t}\right)\right\}_{t \in T}$. Then $\forall t \in T, \pi_{t}: X \rightarrow X_{t}$ is an $M$-fuzzifying convexity preserving function. Moreover, $\mathscr{C}$ is the coarsest $M$-fuzzifying convexity such that $\left\{\pi_{t}: t \in T\right\}$ are $M$-fuzzifying convexity preserving functions.

Proof. Let $t_{0} \in T . \forall B \in 2^{X_{t_{0}}}$, by

$$
\begin{aligned}
\mathscr{C}\left(\left(\pi_{t_{0}}\right)^{-1}(B)\right) & \geq \varphi\left(\left(\pi_{t_{0}}\right)^{-1}(B)\right) \\
& =\bigvee_{t \in T} \bigvee_{\left(\pi_{t}\right)^{-1}(B)=\left(\pi_{t_{0}}\right)^{-1}(B)} \mathscr{C}_{t}(B) \\
& \geq \mathscr{C}_{t_{0}}(B),
\end{aligned}
$$

it implies that $\pi_{t_{0}}: X \rightarrow X_{t_{0}}$ is an $M$-fuzzifying convexity preserving function. By the arbitrariness of $t_{0}$, we know $\forall t \in$ $T, \pi_{t}: X \rightarrow X_{t}$ is an $M$-fuzzifying convexity preserving function. If there is an $M$-fuzzifying convex structure $\mathscr{D}$ on $X$ such that $\forall t \in T, \pi_{t}: X \rightarrow X_{t}$ is an $M$-fuzzifying convexity preserving function, then we need to prove $\mathscr{D} \geq \mathscr{C} . \forall B \in 2^{X}$ and $t \in T$ and if $\left(\pi_{t}\right)^{-1}(G)=B$, then $\mathscr{D}(B)=\mathscr{D}\left(\left(\pi_{t}\right)^{-1}(G)\right) \geq$ $\mathscr{C}_{t}(G)$. Note that $\varphi(B)=\bigvee_{t \in T} \bigvee_{\left(\pi_{t}\right)^{-1}(G)=B} \mathscr{C}_{t}(G)$. We have $\mathscr{D}(B) \geq \varphi(B)$ for all $B \in 2^{X}$. Hence $\mathscr{D} \geq \mathscr{C}$.

Theorem 59. Let $(X, \mathscr{C})$ be the product of $\left\{\left(X_{t}, \mathscr{C}_{t}\right)\right\}_{t \in T}$. Then $\mathscr{C}\left(\prod_{t \in T} A_{t}\right) \geq \bigwedge_{t \in T} \mathscr{C}_{t}\left(A_{t}\right)$.

Proof. Since $\pi_{t}: X \rightarrow X_{t}$ is an $M$-fuzzifying convexity preserving function for each $t \in T$, we know that $\forall A_{t} \in 2^{X_{t}}$, $\mathscr{C}\left(\left(\pi_{t}\right)^{-1}\left(A_{t}\right)\right) \geq \mathscr{C}_{t}\left(A_{t}\right)$. Hence,

$$
\begin{aligned}
\mathscr{C}\left(\prod_{t \in T} A_{t}\right) & =\mathscr{C}\left(\bigcap_{t \in T}\left(\pi_{t}\right)^{-1}\left(A_{t}\right)\right) \\
& \geq \bigwedge_{t \in T} \mathscr{C}\left(\left(\pi_{t}\right)^{-1}\left(A_{t}\right)\right) \\
& \geq \bigwedge_{t \in T} \mathscr{C}_{t}\left(A_{t}\right) .
\end{aligned}
$$

Definition 60. Let $\left\{\left(X, \mathscr{C}_{t}\right)\right\}_{t \in T}$ be a family of $M$-fuzzifying convex structures on $X$. Define a maping $\varphi: 2^{X} \rightarrow M$ by $\varphi(A)=\bigvee_{t \in T} \mathscr{C}_{t}(A)$ for each $A \in 2^{X}$. Then the join of $\left\{\mathscr{C}_{t}\right\}_{t \in T}$, denoted by $\bigsqcup_{t \in T} \mathscr{C}_{t}$, is the one generated by the subbase $\varphi$.
The resulting $M$-fuzzifying convex structure $\left(X, \bigsqcup_{t \in T} \mathscr{C}_{t}\right)$ is called the join of $\left\{\left(X, \mathscr{C}_{t}\right)\right\}_{t \in T}$ and is denoted by $\bigsqcup_{t \in T}\left(X, \mathscr{C}_{t}\right)$.

Theorem 61. Let $\left\{\left(X_{t}, \mathscr{C}_{t}\right)\right\}_{t \in T}$ be a family of $M$-fuzzifying convex structures. Let $X$ be the product of the sets of $X_{t}$ for $t \in T$. Then $\prod_{t \in T}\left(X_{t}, \mathscr{C}_{t}\right)=\bigsqcup_{t \in T}\left(X,\left(\pi_{t}\right)^{-1}\left(\mathscr{C}_{t}\right)\right)$.

Proof. By Theorem 31, we have $\forall A \in 2^{X},\left(\pi_{t}\right)^{-1}\left(\mathscr{C}_{t}\right)(A)=$ $\bigvee\left\{\mathscr{C}_{t}(B):\left(\pi_{t}\right)^{-1}(B)=A\right\}$. Then by the definition of the join, $\varphi$ is a subbase of $\bigsqcup_{t \in T}\left(X,\left(\pi_{t}\right)^{-1}\left(\mathscr{C}_{t}\right)\right)$, where

$$
\varphi(A)=\bigvee_{t \in T}\left(\pi_{t}\right)^{-1}\left(\mathscr{C}_{t}\right)(A)=\bigvee_{t \in T} \bigvee_{\left(\pi_{t}\right)^{-1}(B)=A} \mathscr{C}_{t}(B)
$$

for each $A \in 2^{X}$. By the definition of the product, it is easy to see that $\varphi$ is a subbase of $(X, \mathscr{C})$. The proof is completed.

\section{Conclusion}

In this paper, we introduce a new approach to the fuzzification of convex structures, which is called an $M$-fuzzifying convex structure. This fuzzification is different from these in [3-5]. An $M$-fuzzifying convex structure can be characterized by means of its $M$-fuzzifying closure operator. An $M$-fuzzifying convex structure and its $M$-fuzzifying closure operator are one-to-one corresponding.

In the framework of $M$-fuzzifying convex structure, the concepts of $M$-fuzzifying convexity preserving functions, substructures, disjoint sums, bases, subbases, joins, product, and quotient structures, are presented and their fundamental properties are discussed in $M$-fuzzifying convex structure.

The above facts will be useful to help further investigations and it is possible that the fuzzification of convex structure would be applied to some problems related to the theory of abstract convexity in the future.

\section{Conflict of Interests}

The authors deny that they have any intention to obtain any financial interests.

\section{Acknowledgments}

The project is supported by the National Natural Science Foundation of China (11371002) and the Specialized Research Fund for the Doctoral Program of Higher Education (20131101110048).

\section{References}

[1] M. Berger, "Convexity," The American Mathematical Monthly, vol. 97, no. 8, pp. 650-678, 1990.

[2] M. L. J. van de Vel, Theory of Convex Structures, North-Holland, New York, NY, USA, 1993.

[3] M. V. Rosa, "On fuzzy topology fuzzy convexity spaces and fuzzy local convexity," Fuzzy Sets and Systems, vol. 62, no. 1, pp. 97-100, 1994. 
[4] M. V. Rosa, A study of fuzzy convexity with special reference to separation properties [Ph.D. thesis], Cochin University of Science and Technology, 1994.

[5] Y. Maruyama, "Lattice-valued fuzzy convex geometry," RIMS Kokyuroku, vol. 164, pp. 22-37, 2009.

[6] P. Dwinger, "Characterization of the complete homomorphic images of a completely distributive complete lattice I," Indagationes Mathematicae, vol. 85, pp. 403-414, 1982.

[7] G. J. Wang, "Theory of topological molecular lattices," Fuzzy Sets and Systems, vol. 47, no. 3, pp. 351-376, 1992.

[8] H. Huang and F. Shi, " $L$-fuzzy numbers and their properties," Information Sciences, vol. 178, no. 4, pp. 1141-1151, 2008.

[9] C. V. Negoita and D. A. Ralescu, Applications of Fuzzy Sets to Systems Analysis, Birkhäuser, Basel, Switzerland; Stuttgart and Halsted Press, New York, NY, USA, 1975.

[10] F.-G. Shi, “Theory of $L_{\beta}$-nested sets and $L_{\alpha}$-nested sets and its applications," Fuzzy Systems and Mathematics, vol. 4, pp. 65-72, 1995 (Chinese).

[11] F. G. Shi, "L-fuzzy sets and prime element nested sets," Journal of Mathematical Research and Exposition, vol. 16, no. 3, pp. 398402, 1996 (Chinese).

[12] F.-G. Shi, “Theory of molecular nested sets and its applications," Journal of Yantai Teachers University (Natural Science), vol. 1, pp. 33-36, 1996 (Chinese).

[13] F. Shi, “\$L\$-fuzzy relations and \$L \$-fuzzy subgroups," Journal of Fuzzy Mathematics, vol. 8, no. 2, pp. 491-499, 2000.

[14] W. Kubis, Abstract convex structures in topology and set theory [Ph.D. thesis], University of Silesia, Katowice, Poland, 1999.

[15] F.-G. Shi and B. Pang, "Categories isomorphic to the category of L-fuzzy closure system spaces," Iranian Journal of Fuzzy Systems, vol. 10, no. 5, pp. 127-146, 2013.

[16] M.-S. Ying, "A new approach for fuzzy topology (I)," Fuzzy Sets and Systems, vol. 39, no. 3, pp. 303-321, 1991.

[17] A. K. Katsaras and D. B. Liu, "Fuzzy vector spaces and fuzzy topological vector spaces," Journal of Mathematical Analysis and Applications, vol. 58, no. 1, pp. 135-146, 1977.

[18] J. Fang and P. Chen, "One-to-one correspondence between fuzzifying topologies and fuzzy preorders," Fuzzy Sets and Systems, vol. 158, no. 16, pp. 1814-1822, 2007. 


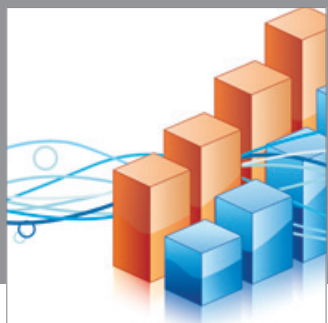

Advances in

Operations Research

mansans

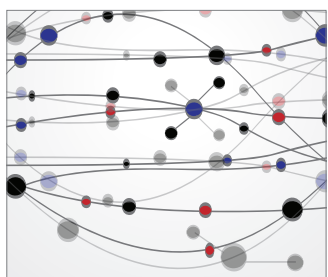

The Scientific World Journal
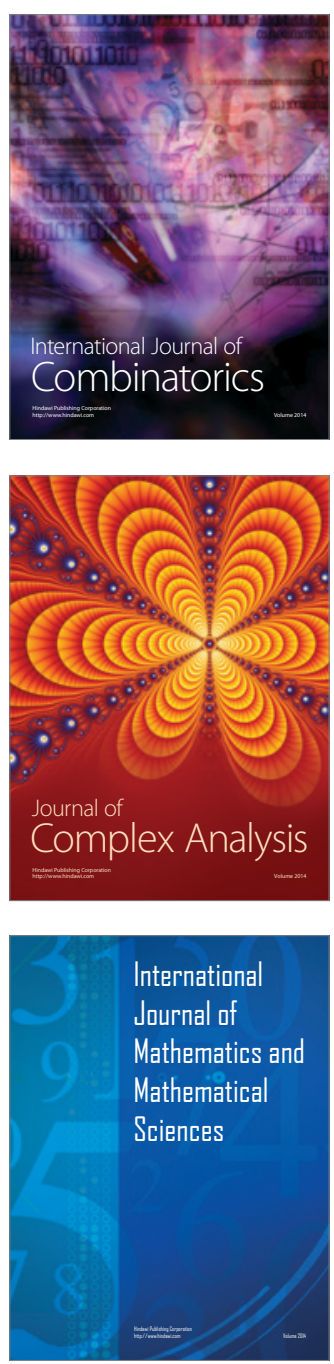
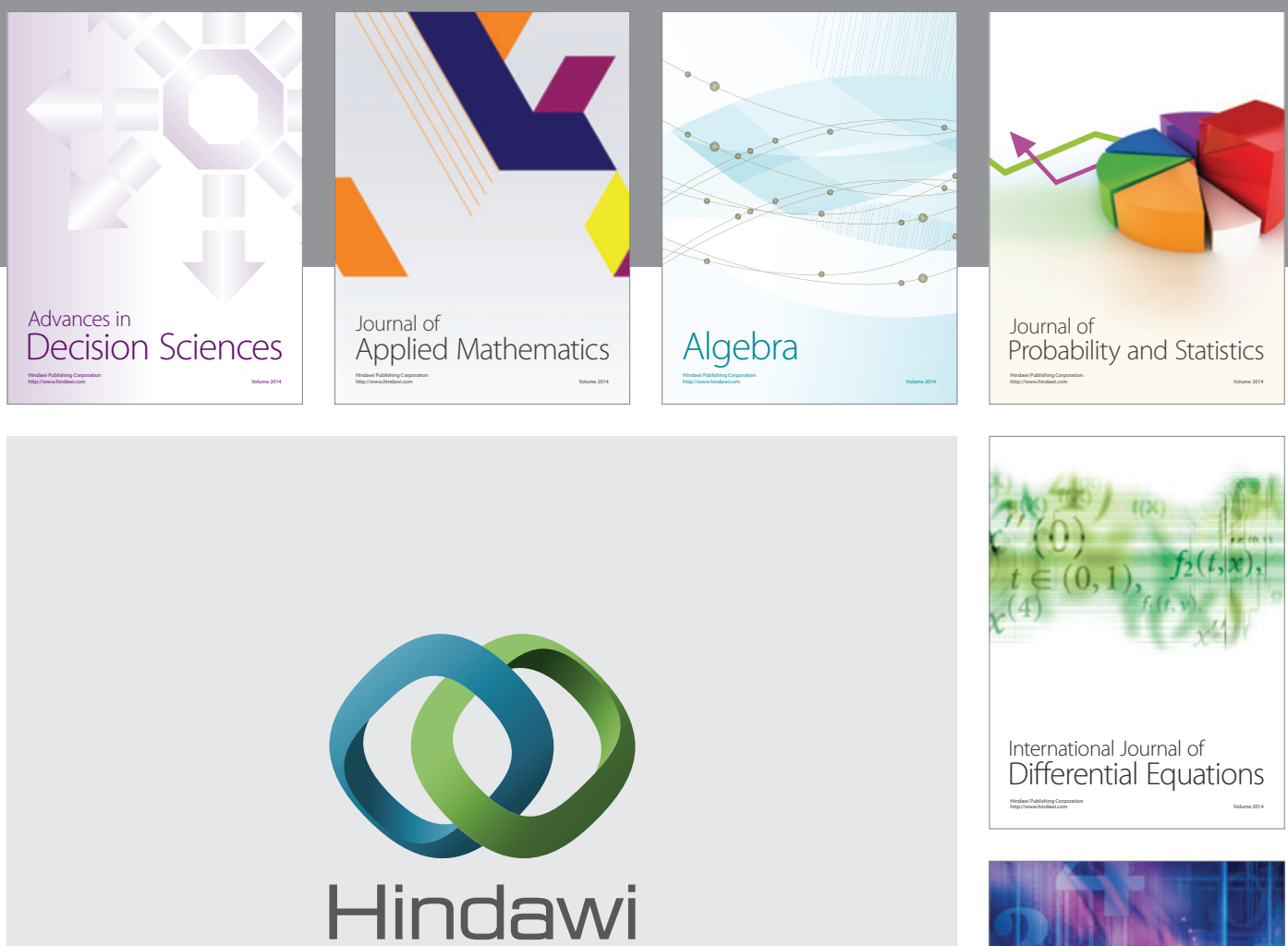

Submit your manuscripts at http://www.hindawi.com
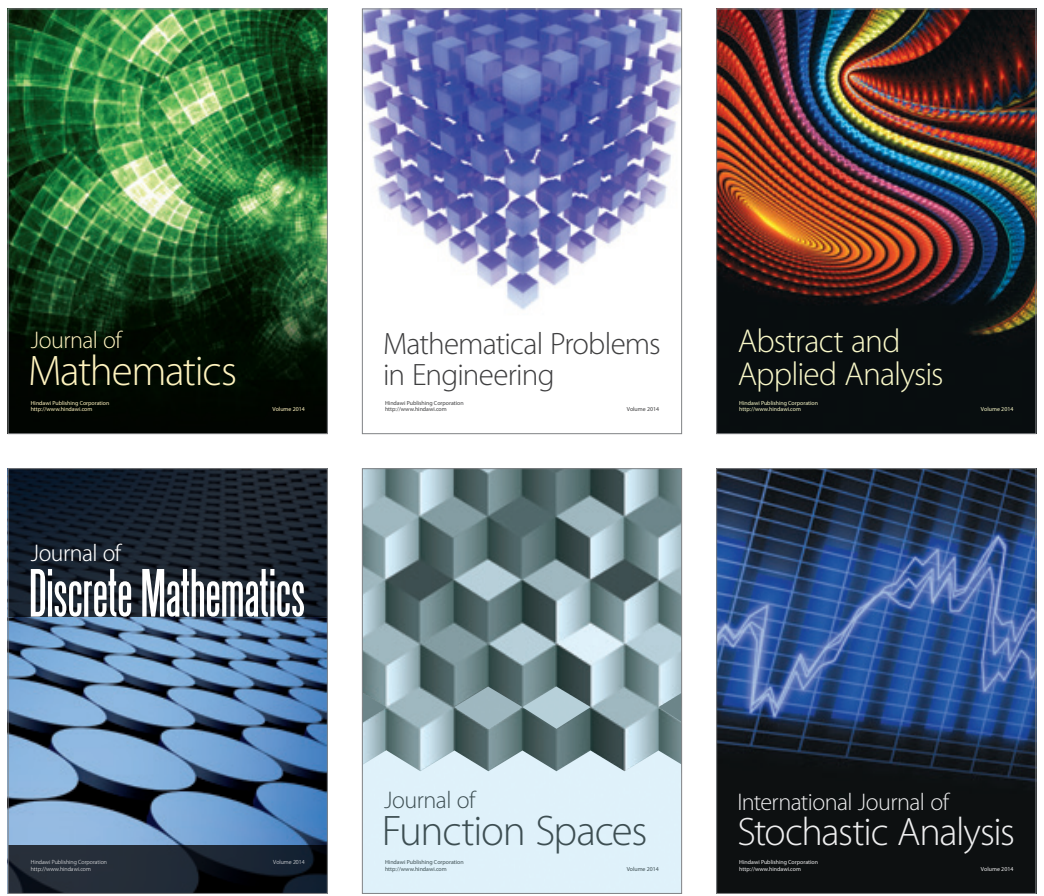

Journal of

Function Spaces




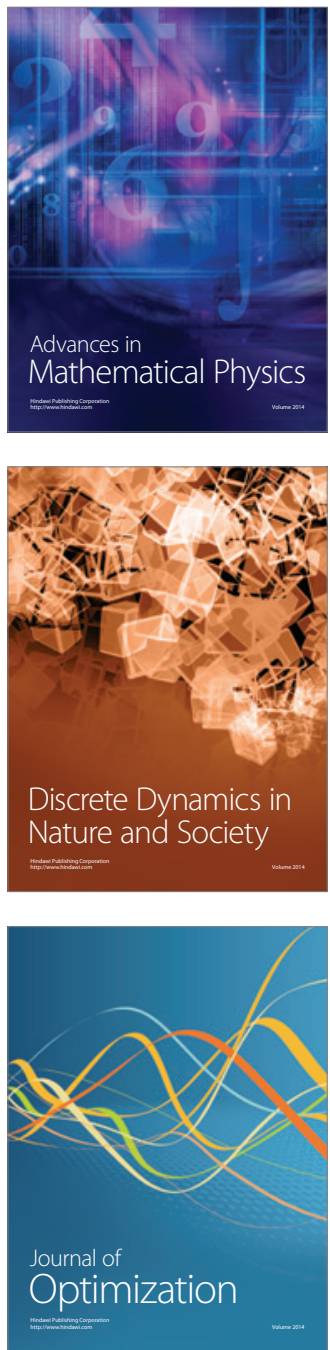